\title{
SDR9C7 catalyzes critical dehydrogenation of acylceramides for skin barrier formation
}

\author{
Takuya Takeichi, ${ }^{1}$ Tetsuya Hirabayashi, ${ }^{2}$ Yuki Miyasaka, ${ }^{3}$ Akane Kawamoto, ${ }^{4}$ Yusuke Okuno, ${ }^{5}$ Shijima Taguchi, ${ }^{6}$ Kana Tanahashi, \\ Chiaki Murase, ${ }^{1}$ Hiroyuki Takama, ${ }^{7}$ Kosei Tanaka, ${ }^{8}$ William E. Boeglin, ${ }^{9}$ M. Wade Calcutt, ${ }^{10}$ Daisuke Watanabe, ${ }^{7}$ Michihiro Kono, ${ }^{1}$ \\ Yoshinao Muro, ${ }^{1}$ Junko Ishikawa, ${ }^{4}$ Tamio Ohno, ${ }^{3}$ Alan R. Brash, ${ }^{9}$ and Masashi Akiyama ${ }^{1}$ \\ 'Department of Dermatology, Nagoya University Graduate School of Medicine, Nagoya, Japan. ${ }^{2}$ Laboratory of Biomembrane, Tokyo Metropolitan Institute of Medical Science, Tokyo, Japan. ${ }^{3}$ Division of \\ Experimental Animals, Nagoya University Graduate School of Medicine, Nagoya, Japan. ${ }^{4}$ Biological Science Research Laboratories, Kao Corporation, Haga, Tochigi, Japan. ${ }^{5}$ Medical Genomics Center, Nagoya \\ University Hospital, Nagoya, Japan. ${ }^{6}$ Division of Dermatology, Mito Kyodo General Hospital, Mito, Ibaraki, Japan. ${ }^{7}$ Department of Dermatology, Aichi Medical University, Nagakute, Japan. ${ }^{8}$ Analytical Science \\ Research Laboratories, Kao Corporation, Haga, Tochigi, Japan. ${ }^{9}$ Departments of Pharmacology and Biochemistry and ${ }^{10}$ Vanderbilt Institute of Chemical Biology, Vanderbilt University, Nashville, Tennessee, USA.
}

\begin{abstract}
The corneocyte lipid envelope, composed of covalently bound ceramides and fatty acids, is important to the integrity of the permeability barrier in the stratum corneum, and its absence is a prime structural defect in various skin diseases associated with defective skin barrier function. SDR9C7 encodes a short-chain dehydrogenase/reductase family 9C member 7 (SDR9C7) recently found mutated in ichthyosis. In a patient with SDR9C7 mutation and a mouse Sdr9c7-KO model, we show loss of covalent binding of epidermal ceramides to protein, a structural fault in the barrier. For reasons unresolved, protein binding requires lipoxygenase-catalyzed transformations of linoleic acid (18:2) esterified in $\omega$ - 0 -acylceramides. In $S d r 9 c 7^{-/-}$epidermis, quantitative liquid chromatography-mass spectometry (LC-MS) assays revealed almost complete loss of a species of $\omega$ - 0 acylceramide esterified with linoleate-9,10-trans-epoxy-11E-13-ketone; other acylceramides related to the lipoxygenase pathway were in higher abundance. Recombinant SDR9C7 catalyzed NAD+-dependent dehydrogenation of linoleate 9,10-trans-epoxy-11E-13-alcohol to the corresponding 13-ketone, while ichthyosis mutants were inactive. We propose, therefore, that the critical requirement for lipoxygenases and SDR9C7 is in producing acylceramide containing the 9,10-epoxy$11 E-13-k e t o n e$, a reactive moiety known for its nonenzymatic coupling to protein. This suggests a mechanism for coupling of ceramide to protein and provides important insights into skin barrier formation and pathogenesis.
\end{abstract}

\section{Introduction}

During the evolutionary process, when our ancestors left the aquatic environment, our integument developed a robust protective structure in the uppermost epidermis, the stratum corneum, and adjusted to the dry environment on the ground (1). The water barrier property of the stratum corneum is achieved by a fusion of lipids and proteins in the terminally differentiated corneocytes of the outer epidermis; the cell membrane is replaced by a coat of cross-linked proteins (the cornified cell envelope, CCE), and multilaminar lipid layers mainly composed of ceramides, cholesterol, and free fatty acids fill the space between the cells. Interfacing between the two on the outside surface of the CCE is a layer of covalently bound ceramides and fatty acids, the corneocyte lipid envelope (CLE) (2-4). The importance of the CLE to the integrity of the permeability barrier is known from its absence being a prime structural defect in many diseases of barrier function $(4,5)$. Among the epidermal ceramides, a key component is the $\omega$-O-acylceramide CerEOS (esterified omega-hydroxy sphingosine). Defects in genes involved in biosynthesis of CerEOS or its processing to form the CLE result in the severe skin disorder

Conflict of interest: The authors have declared that no conflict of interest exists. Copyright: (c) 2020, American Society for Clinical Investigation.

Submitted: May 29, 2019; Accepted: October 24, 2019; Published: January 13, 2020.

Reference information: / Clin Invest. 2020;130(2):890-903.

https://doi.org/10.1172/JCI130675. autosomal recessive congenital ichthyosis (ARCI), characterized by dry skin, scaling, hyperkeratosis, and occasional erythroderma $(6,7)$. Moreover, alterations of epidermal lipid homeostasis, especially contents of CerEOS and other ceramides, are linked to atopic dermatitis (8). Thus, abnormal metabolism of epidermal ceramides underlies the pathogenesis of various skin diseases associated with defective skin barrier function.

The esterified component of CerEOS is the essential fatty acid linoleate $(\mathrm{C} 18: 2 \omega 6)$, linked through an ester bond to the eponymous fatty acid omega-hydroxyl of EOS (9). Enzymatic oxidation of the linoleate ester in CerEOS by lipoxygenases (LOXs) is required for formation of the CLE $(10,11)$. Loss-offunction of $12 R$-lipoxygenase $(12 R-\mathrm{LOX})$ or epidermal lipoxygenase-3 (eLOX3) by gene mutations results in impaired permeability barrier function in patients with ARCI and in the corresponding knockout mice (12-14). The linoleate moiety of EOS cannot be replaced functionally by other fatty acids because they do not serve as a substrate for these epidermal LOXs. Accordingly, substitution of linoleate by oleate in CerEOS by prolonged intake of a diet lacking essential fatty acids impairs the normal formation of protein-bound ceramides (15), leading to an ichthyosis-like condition with dermatitis and increased transepidermal water loss (TEWL).

The mechanism in which the CLE is constituted from oxidized EOS on the surface of CCE has not been fully resolved. It has been 
assumed that an unidentified esterase/lipase de-esterifies the oxidized linoleate in EOS to generate free $\omega$-hydroxy ceramide (CerOS) for covalent binding to the CCE, thus forming the CLE (4, $5,10,11,16)$. Transglutaminase could catalyze the covalent attachment of the free $\omega$-OH group to glutamate or glutamine residues of CCE proteins such as involucrin, periplakin, and envoplakin $(17,18)$. However, the transglutaminase(s) are not identified, and known gene knockouts do not exhibit the expected skin phenotype with absence of the CLE $(19,20)$. Furthermore, results inconsistent with the current line of thinking come from inactivation studies with PNPLA1, the gene that esterifies linoleate to CerOS, forming CerEOS (21-25). In Pnpla1-deficient mice or patients with mutations in the PNPLA1 gene, the CLE is almost absent even though CerOS and its glucosylated form are highly accumulated (22, 24, 25). This suggests that the prerequisite for lipoxygenase-catalyzed oxidation of CerEOS is not in order to facilitate de-esterification of the linoleate moiety and produce CerOS, but rather has a different functional role during formation of the CLE.

Herein we report what we believe are new insights into skin barrier formation stemming from an understanding of the activities of a recently described ARCI gene, SDR9C7. SDR9C7 encodes a shortchain dehydrogenase/reductase family 9C member 7 (SDR9C7) (26, 27), and is highly expressed in the epidermis (28). In 2016, 2 missense mutations in SDR9C7 were identified in patients with ARCI (29), and several more in 2018 (30). Our ultrastructural observations of the skin lesion of an ARCI patient with SDR9C7 mutations revealed disruption of the intercellular lipid layers in the stratum corneum (31). Now we have unraveled the pathomechanism and the enzymology of SDR9C7 through detailed structural and biochemical analyses in a patient's epidermis, by development and characterization of an $S d r 9 c 7$ knockout mouse model, and by correlating these findings with the catalytic properties of the recombinant SDR9C7 enzyme. We clarify the biochemical function of SDR9C7 as catalyzing an oxidation of the lipoxygenase products esterified in CerEOS. This generates a chemically reactive epoxy-enone derivative that has the ability to bind directly to protein. This bypasses the need for removal of the oxidized linoleate from CerEOS prior to covalent binding. Thus, our results indicate an unsuspected role of SDR9C7 in facilitating covalent binding of oxidized acylceramide to CCE proteins, thus forming the CLE, the key to structural integrity of the permeability barrier of the epidermis.

\section{Results}

Loss of the CLE in a patient with a recessive SDR9C7 mutation. We identified a mutation in a patient with clear ARCI symptomatology (Figure 1A and Supplemental Figure 1, A and B; supplemental material available online with this article; https://doi.org/10.1172/ JCI130675DS1) as a rare homozygous missense mutation in the SDR9C7 gene (c.826C>T, p.Arg276Cys, rs755404310), as confirmed by Sanger sequencing (Supplemental Figure 1C); only 3 heterozygous carriers are reported so far in the gnomAD database (32). Whole-exome analysis showed no mutations in other genes previously implicated in the molecular pathology of ichthyosis and the R276C mutation in recombinant human SDR9C7 results in complete loss of catalytic activity (see below).

A skin biopsy specimen showed compact hyperkeratosis with a normal-appearing granular layer (Figure 1B). Immunohistochem- ical analysis showed low immunoreactivity for SDR9C7 in the stratum corneum and granulosum in a lesional skin sample from the patient compared with control (Figure 1C), suggesting that SDR9C7 with the R276C mutation results either in lower transcription or in an unstable protein that is degraded rapidly in the differentiated keratinocytes. Lipids in the tape-stripped skin samples from the patient, her unaffected parents, and normal controls were assessed by LC-MS and thin-layer chromatography. Although the overall effects of bi-allelic mutation on the levels of total ceramide, nonesterified ceramides, and free fatty acids were small, notably, the EOS levels in the patient's epidermis are 2.8-fold elevated compared with normal controls (Figure 1, D-G, and Supplemental Tables 1-8). Mechanistically tied to this increase in CerEOS is the almost complete loss of the covalently bound ceramides CerOS and $\mathrm{CerOH}$ in the patient (Figure 1, $\mathrm{H}$ and I; Table 1 and Table 2). These results suggest that functional SDR9C7 is required for formation of the fundamental structural component of the epidermal permeability barrier, the CLE, from its main precursor CerEOS.

$S d r 9 c 7^{-1}$ mice recapitulate aspects of the human ichthyosis. To gain deeper insight into the function of Sdr9c7 in vivo, we used a CRISPR-Cas9 gene-targeting approach to generate $S d r 9 c 7$ knockout mice. The frameshift mutation in the $S d r 9 c 7$ gene (Supplemental Figure 2, A and B) likely leads to nonsense-mediated decay of the mRNA transcript (Supplemental Figure 3E). The expression of $\mathrm{Sdr} 9 \mathrm{c7}$ protein in $S d r 9 c 7^{-/-}$epidermis was also not detected by immunohistochemistry with a polyclonal antibody to the Cterminal region of Sdr9c7 (Supplemental Figure 2C). Offspring from heterozygote intercrosses were born at the expected Mendelian ratio (Supplemental Table 9). Although the $S d r 9 c 7^{+/-}$mice were healthy and indistinguishable from WT mice, newborn $S d r 9 c 7^{-1-}$ pups had dry skin (Figure 2A) and died within 5 hours after birth. The $S d r 9 c 7^{-/-}$pups were lighter at birth than the $S d r 9 c 7^{+/+}$and $S d r 9 c 7^{+/-}$genotypes (about 70\%,P<0.01), and they lost additional weight over 5 hours (Supplemental Figure 2D). In accordance with this steep weight reduction, TEWL was markedly higher in the $S d r 9 c 7^{-/-}$newborns (Figure 2B), indicating a severe defect of the inside-out barrier in the null mice. This was supported by a toluidine blue exclusion assay in which the WT littermates excluded dye, whereas $S d r 9 c 7^{-1-}$ pups showed robust dye penetration into the skin (Figure 2C).

As expected, histological analysis revealed that $S d r 9 c 7^{+/+}$mice had a clear basket weave-like structure segregated by interspaces, indicative of the presence of lipid lamellae (Figure 2D, left). In contrast, $S d r 9 c 7^{-/-}$mice exhibited a tightly packed structure in the stratum corneum, a reduced number of keratohyaline granules in the uppermost stratum granulosum, and epidermal hyperplasia (Figure 2D, right), which is an adaptive response to barrier disruption. These findings are consistent with histological features in the skin sample from the ARCI patient with the SDR9C7 mutation (Figure 1B). Ultrastructurally, in the stratum corneum of $S d r 9 c 7^{-/-}$ mice, CLE was defective or completely lacking, compared with the intact CLE in the stratum corneum of WT mice (Figure 2, E and F, and Supplemental Figure 2E). The absence of protein products from $S d r 9 c 7$ in the skin of $S d r 9 c 7^{-/-}$mice was confirmed by immunohistochemistry (Supplemental Figure 2C). Moreover, LC-MS analysis of the covalently bound CerOS from the protein pellets from $S d r 9 c 7^{+/+}$and $S d r 9 c 7^{-/-}$skin samples revealed a nearly 
A

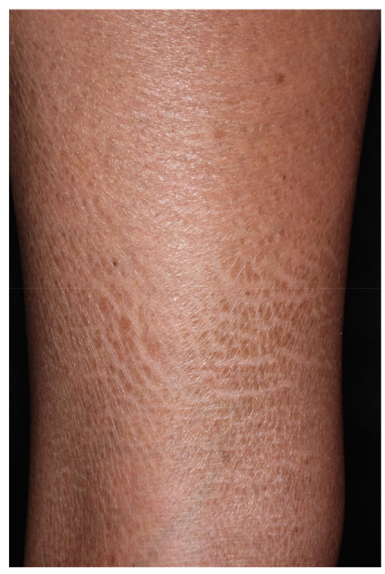

B

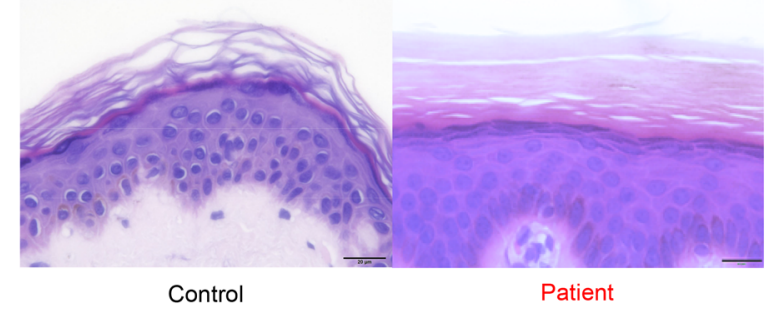

C

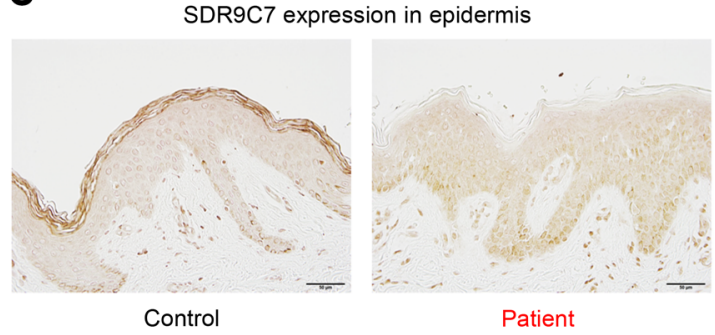

E

D

Total ceramides in upper arm
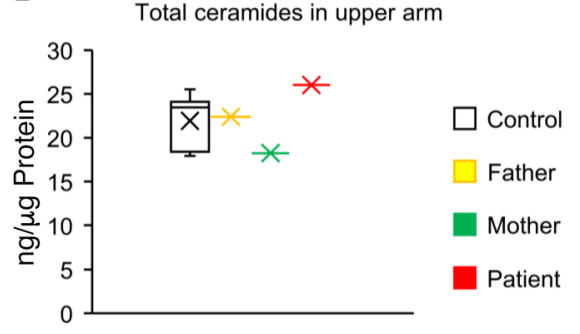

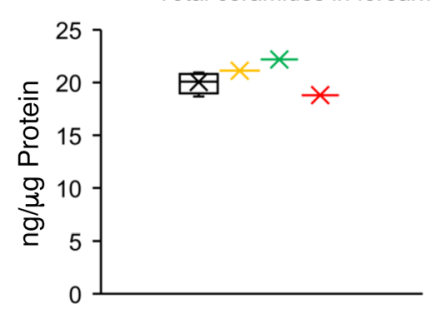

Figure 1. Changes in SDR9C7 expression and major changes in ceramides in the patient's skin. (A) Thin shiny scales on the lower leg are observed. (B) A biopsy sample from the ichthyotic skin of the proband shows marked compact hyperkeratosis, which suggests deficiency of the intercellular lipid layers in the stratum corneum. In contrast, the stratum corneum of the normal control skin shows a basket-weave pattern. Scale bars $=20 \mu \mathrm{m}$. (C) Immunohistochemistry of the lesional skin with an anti-SDR9C7 antibody. The staining intensity is strongly reduced in the patient's skin compared with the skin from a healthy control. Scale bars $=50 \mu \mathrm{m}$. ( $\mathbf{D}$ and $\mathbf{E}$ ) Total ceramide levels in tapestripped samples of epidermis are within the normal range in the patient compared with her parents and the normal controls $(n=8)$. Data represent mean \pm SEM. (F and $\mathbf{G )}$ Individual ceramide species showed minor differences in the patient's forearm and upper arm, with the notable exception of CerEOS, $200 \%-280 \%$ elevated in the patient's epidermis. (H and I) By contrast, ceramides covalently bound to protein were severely reduced in the patient's skin compared with the parents and controls $(n=8)$. Data represent mean \pm SEM.
$\mathbf{F}$

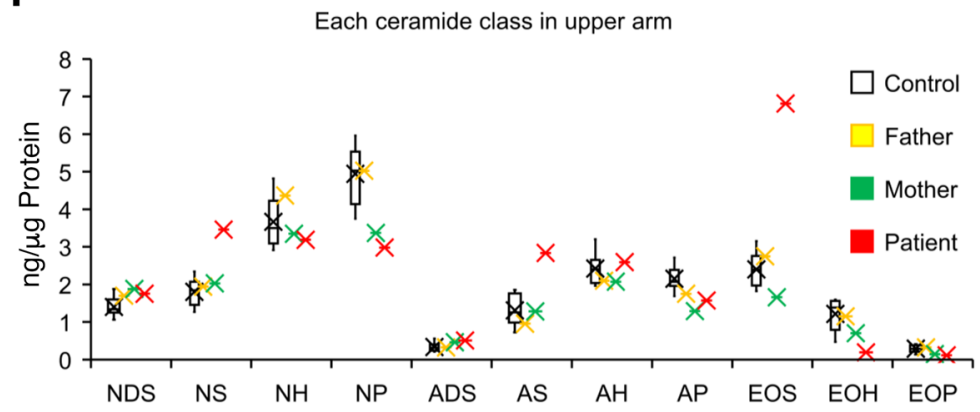

G

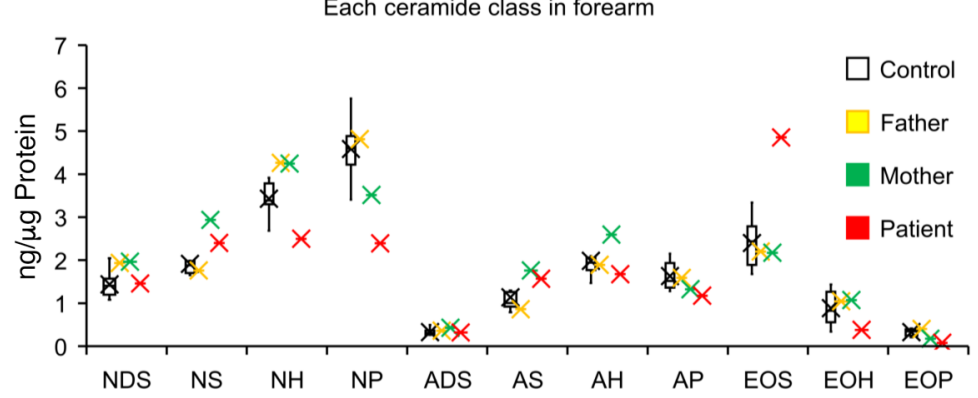

\section{H}

Covalently bound ceramides in upper arm

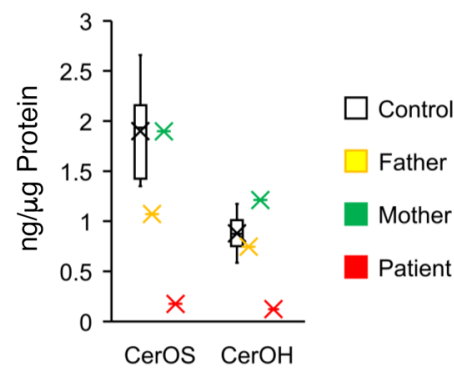

I

Covalently bound ceramides in forearm

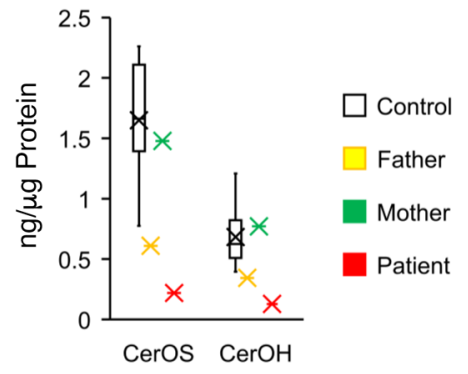

90\% reduction of bound CerOS in the $S d r 9 c 7$ knockout epidermis (Figure 2G). These phenotypes, which have been commonly observed in mutant mice with disruption of genes associated with ARCI caused by skin barrier defects, indicate that $\mathrm{Sdr} 9 \mathrm{c} 7$ is required for epidermal permeability barrier function.
Gene expression profile in the skin of Sdr9c7/- mice. We performed microarray gene profiling using skins of newborn $S d r 9 c 7^{7^{++}}$ and $S d r 9 c 7^{-/}$mice, and focused on differentially expressed genes (DEGs) associated with the following key words related to skin barrier function and ichthyoses: ichthyosis, acylceramide metabolism, 
Table 1. Amounts of covalently bound ceramides in the stratum corneum of the upper arm from the patient and her parents: comparison with those of normal controls

$\begin{array}{lcccc}\text { Total }(\mu \mathrm{g} / \mu \mathrm{g} \text { protein) } & \text { Patient (p.Arg276Cys homo) } & \text { Father (p.Arg276Cys hetero) } & \text { Mother (p.Arg276Cys hetero) } & \text { Controls, } \boldsymbol{n = 8} \text { (mean } \pm \text { SD) } \\ \text { CerOS } & 0.18 & 1.07 & 1.90 & 1.90 \pm 0.44 \\ \text { CerOH } & 0.12 & 0.75 & 1.21 & 0.88 \pm 0.18\end{array}$

retinoid metabolism, steroid metabolism, and keratinocyte differentiation. There was no significant change in the expression of most genes related to the retinoid metabolism, steroid metabolism, and keratinocyte differentiation (Supplemental Figure 3D). Real-time quantitative PCR (qPCR) confirmed the slightly diminished expression of Aloxe 3 in the $S d r 9 c 7^{-/}$skin relative to the WT skin, whereas mRNA expression levels of the Alox12b, Tgm1, and Lor were similar between the 2 genotypes (Supplemental Figure 3E). Additionally, the $S d r 9 c 7^{-1-}$ skin showed a marked reduction in expression of Irf4, a transcription factor required for functional development of type 1 regulatory T cells (33), and interpreted as evidence of secondary and global disruptions in epidermal functionality.

We also compared gene expression profiles between mouse lines deficient in $S d r 9 c 7$ and Pnpla1; the latter selectively utilizes linoleic acid for acylceramide biosynthesis, and loss of this activity causes severe epidermal barrier defects (22). Overall, the gene expression changes in the skin from $S d r 9 c 7^{-/-}$mice were smaller compared with Pnpla1 ${ }^{-/}$mice (Supplemental Figure 3, A and B). Venn diagrams and heatmaps indicated that the number of overlapping DEGs in these 2 knockout lines was very limited (Supplemental Figure 3, C and D. For further details, see the legend of Supplemental Figure 3). Most significantly, whereas gene expression associated with the biosynthesis of acylceramides (Elovl4, Cyp4f39, Cers3, and Abhd5) was increased in Pnpla1 ${ }^{-1-}$ skin as shown previously (22), there were no similar changes in $S d r 9 c 7^{-/-}$ skin (Supplemental Figure 3D). These results suggest that the deficiency of Sdr9c7 possibly affects the processes after the production of acylceramides, not the differentiation itself of keratinocytes in the epidermis.

Sdr9c7 knockout induces major changes in epidermal oxidized ceramides. TLC analysis of epidermal lipids extracted from neonatal WT and $S d r 9 c 7^{-1-}$ mice revealed accumulation of a prominent and unknown lipid in the $S d r 9 c 7^{-1-}$ knockout epidermis (Figure 3A). The new band appears between the polarities of authentic standards of CerOS and acylglucosylceramide (Glc-CerEOS), and is not seen in the skins of $S d r 9 c 7^{+/+}, S d r 9 c 7^{+/}$, and Pnpla1 $1^{-/-} /\left(S d r 9 c 7^{7^{++}}\right)$mice. The Pnpla1 ${ }^{-/}$animals act as a significant control because they are deficient in the coupling of linoleic acid into CerEOS (22), the band of which is missing in the Pnpla1 $1^{-/}$lane(s) of the TLC plate (Figure
3A). The $S d r 9 c 7^{-/}$skins, by contrast, show a normal appearance of CerEOS, indicating its biosynthesis is intact and suggesting any defects in metabolism occur beyond the step of EOS formation in the processing of the epidermal ceramides.

The enzymology of epidermal CLE construction includes the lipoxygenase-catalyzed oxidation of CerEOS through the action of $12 R$-LOX and eLOX-3 (10). To investigate the possible involvement of SDR9C7 in this pathway, we examined the epidermis from neonatal WT and $S d r 9 c 7^{--}$pups for LOX products by qualitative (product screening) and quantitative LC-MS. Screening by normalphase LC-MS revealed the appearance of a prominent new polar product in $S d r 9 c^{-/-}$epidermis, one that was undetectable by this methodology in the WT, and in accord with its polarity on TLC, the new peak chromatographed on normal-phase HPLC between EOS and Glc-CerEOS (Figure 3B). The molecular masses of all the main peaks on the chromatograms were easily detectable by APCI mass spectrometry, thus identifying the WT profile and the corresponding peaks in the knockout as typical epidermal ceramides (Supplemental Figures 4-15), and establishing the chemical structure of the prominent new peak in the $S d r 9 c 7^{-1-}$ epidermis. The main species of the new polar product showed a $\mathrm{MH}^{+}$ion at $\mathrm{m} / z$ 1117, compatible with CerEOS containing a C34:1 $\omega$-hydroxy-ultra longchain fatty acid ( $\omega$-OH-ULCFA) esterified with a trihydroxy-C18:1 fatty acid (Figure 3C). This was confirmed by negative ion detection of $m / z 329$, corresponding to the mass of a trihydroxy-C18:1 fatty acid (Supplemental Figure 12), as well as positive ion detection of product ions characteristic of a C18:1 sphingosine moiety (Supplemental Figure 13) (34). Thus, the intense new product in the $S d r 9 c 7$ knockout epidermis was identified as CerEOS esterified with trihydroxy-C18:1 (EOS-triol). Moreover, a degraded derivative of EOS-triol lacking the sphingosine moiety, namely $\omega$-OH-ULCFA esterified with C18:1-triol was also detected in the $S d r 9 c 7^{-/-}$epidermis (Supplemental Figures 8 and 9).

To further explore the influence of Sdr9c7 deficiency on the lipoxygenase pathway derivatives of CerEOS, epidermal lipids from WT and $S d r 9 c 7^{-/-}$knockout mice were analyzed by quantitative LC-MS (Figure 4, A and B). The most striking finding, confirming the results above, is the astonishing 100-fold elevation over WT of EOS-triol in the $S d r 9 c 7^{-/}$epidermis (Figure 4, A and B).

Table 2. Amounts of covalently bound ceramides in the stratum corneum of the forearm from the patient and her parents: comparison with those of normal controls

$\begin{array}{lcccc}\text { Total }(\mu \mathrm{g} / \mu \mathrm{g} \text { protein) } & \text { Patient (p.Arg276Cys homo) } & \text { Father (p.Arg276Cys hetero) } & \text { Mother (p.Arg276Cys hetero) } & \text { Controls, } \boldsymbol{n}=\mathbf{8} \text { (mean } \pm \text { SD) } \\ \text { CerOS } & 0.22 & 0.61 & 1.48 & 1.65 \pm 0.48 \\ \text { CerOH } & 0.13 & 0.34 & 0.77 & 0.68 \pm 0.26\end{array}$


A

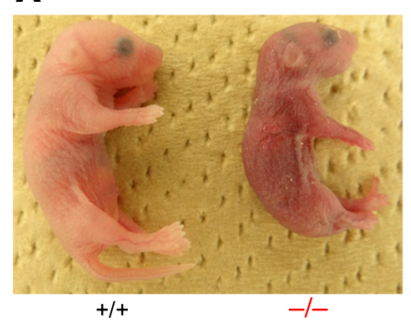

C

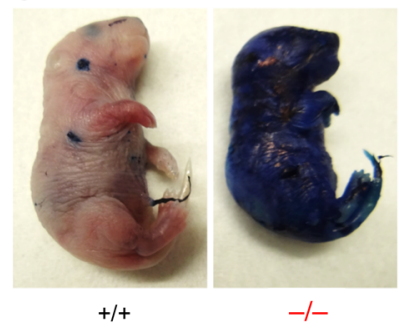

$\mathbf{E}$

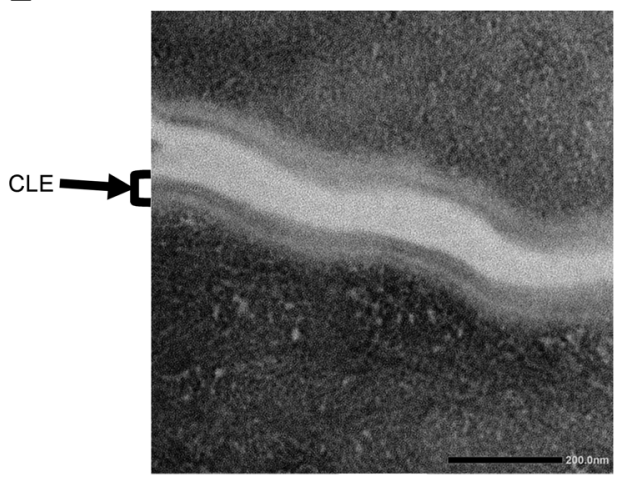

$+/+$

B

D
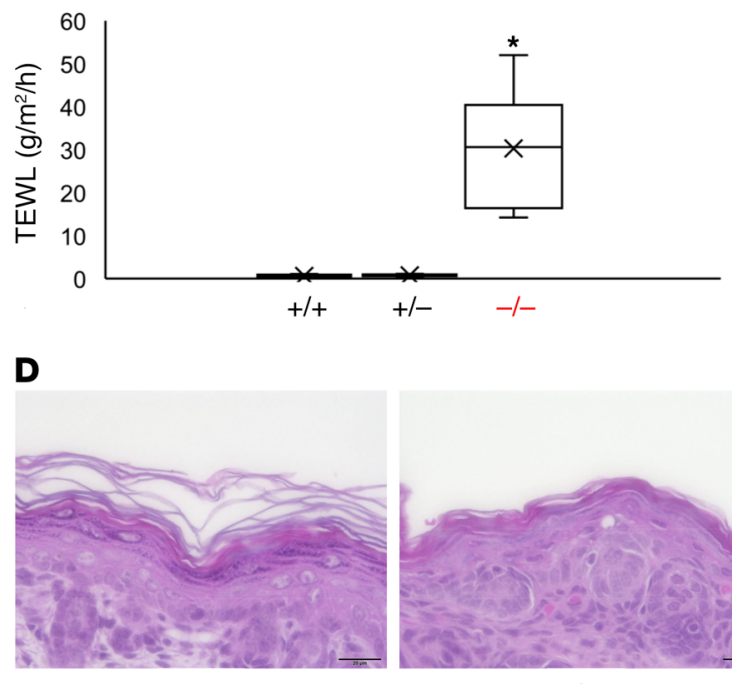

$+/+$

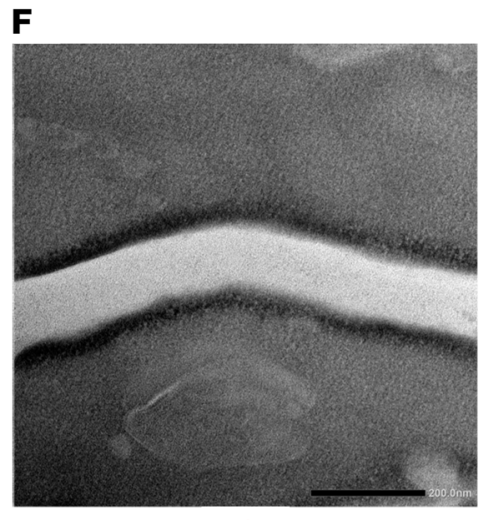

G

Covalently bound CerOS (\% of WT)

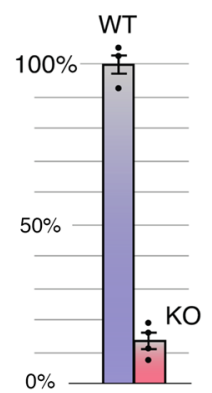

Figure 2. Sdr9c7 deletion reduces permeability barrier function and CLE formation. (A) Gross appearance of $S d r 9 c 7^{+/+}$and $S d r 9 c 7^{-/-}$newborns at 1 hour after birth. Although the newborns ( $S d r 9 c 7^{+/+}, S d r 9 c 7^{+--}, S d r 9 c 7^{-1-}$ ) were indistinguishable at birth, Sdr9c $7^{-1-}$ mice show erythema and wrinkled skin consistent with increased TEWL soon after birth. (B) Skin permeability as assessed by TEWL on the dorsal skin surface of $S d r 9 c 7^{+/+}(n=6), S d r 9 c 7^{+/-}(n=7)$, and $S d r 9 c 7^{-l_{-}}(n=10)$ mice (mean \pm SEM, ${ }^{*} P<0.001$ ). (C) Toluidine blue exclusion assay using neonatal Sdr9c $7^{+/+}$and $S d r 9 c 7^{-1-}$ mice. (D) Histology of dorsal skin sections from new-

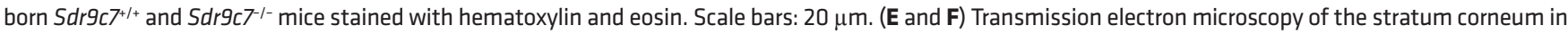
$S d r 9 c 7^{+/+}$and $S d r 9 c 7^{-1-}$ newborn mice. Scale bars = $200 \mu \mathrm{m}$. (E) There is a normal external lipid membrane monolayer, the corneocyte-bound lipid envelope (CLE),

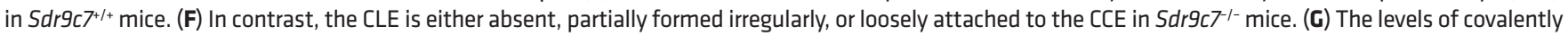

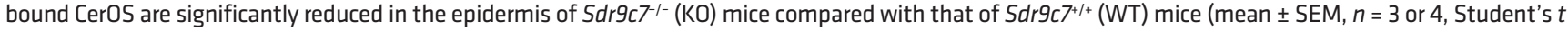
test, $P<0.001)$.

The quantitative LC-MS analysis identified this hugely elevated linoleate-derived triol as specifically the $9 R, 10 S, 13 R$-trihydroxy isomer (Figure $4 \mathrm{C}$ ), the one formed selectively via the consecutive actions of $12 R$-LOX, eLOX3, and epoxide hydrolase $(34,35)$.

The most prominent oxidized linoleate esterified to CerEOS in the WT epidermis is the epoxy-ketone (Figure 4, A and B), and this 9,10-epoxy-11E-13-ketone is largely eliminated in the $S d r 9 c 7^{-1-}$ epidermis (only 7\% of WT) (Figure 4, A, B, and D). On account of SDR9C7 being a member of the short-chain dehydrogenase/ reductase family, its expected direct activity is in oxidation of lipids containing an alcohol group adjacent to a double bond, producing the corresponding allylic ketone. This activity equates with oxidation of the $12 R$-LOX/eLOX3 product 9,10-epoxy-11E13-alcohol into the 9,10-epoxy-11E-13-ketone, and is consistent with drastic reduction of the epoxy-ketone in the $S d r 9 c 7$ knockout epidermis. In further support of this scenario, the knockout epidermis contains 10-fold higher abundance of the putative Sdr9c7 substrate, the 9,10-epoxy-11E-13-alcohol (Epoxy-OH in Figure 4, $\mathrm{A}$ and $\mathrm{B})$. 9-HODE, the further upstream pathway product, is also elevated in the knockout (Figure 4, A and B). And as presented earlier, unmetabolized CerEOS accumulated in the epidermis of the ichthyosis patient with the SDR9C7 mutation (Figure 1). Taken together, the ceramide analyses point to the primary catalytic activity of SDR9C7 being the oxidation of epoxy-alcohol esterified in CerEOS to the corresponding epoxy-ketone.

As indicated in the Introduction, the ultimate structural defect in the knockout of many ARCI genes is a lack of covalently bound ceramides in the epidermal barrier. For example, this is a proven consequence in deficiencies of PNPLA1, 12R-LOX, eLOX3, and other ARCI genes $(4,5)$. And, as indicated earlier, analysis of the covalently 
A

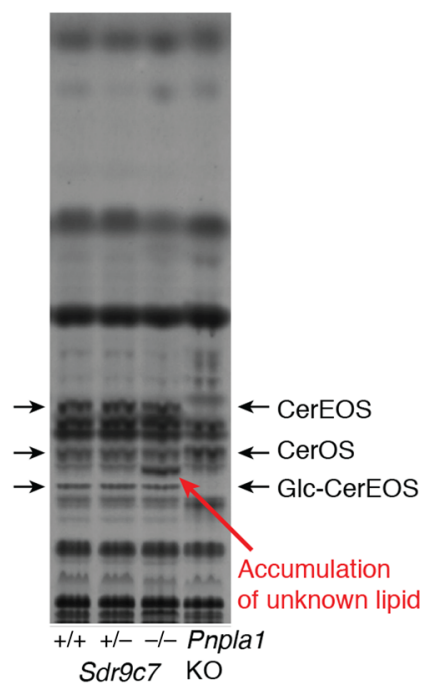

B
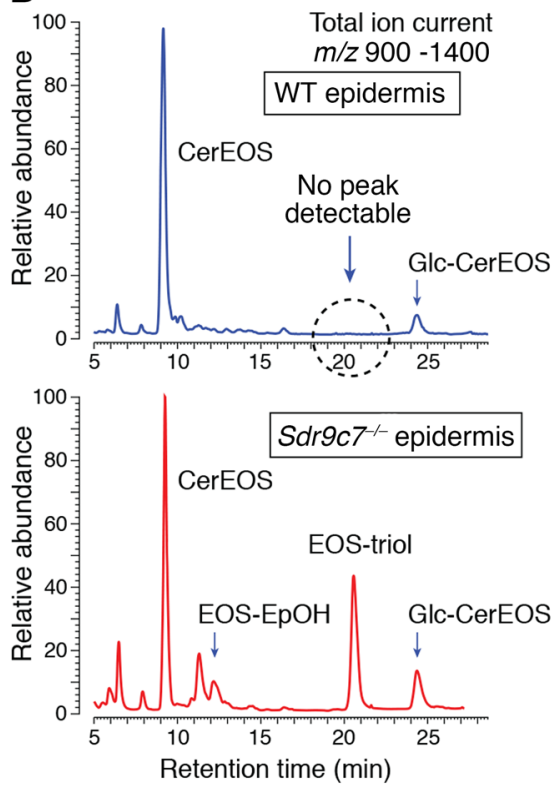

c

CerEOS-triol (with $\omega$-hydroxy-34:1 ULCFA)

Chemical formula: $\mathrm{C}_{70} \mathrm{H}_{133} \mathrm{O}_{8} \mathrm{~N}$

Exact mass: $1116.00, \mathrm{MH}^{+} \mathrm{m} / \mathrm{z} 1117$
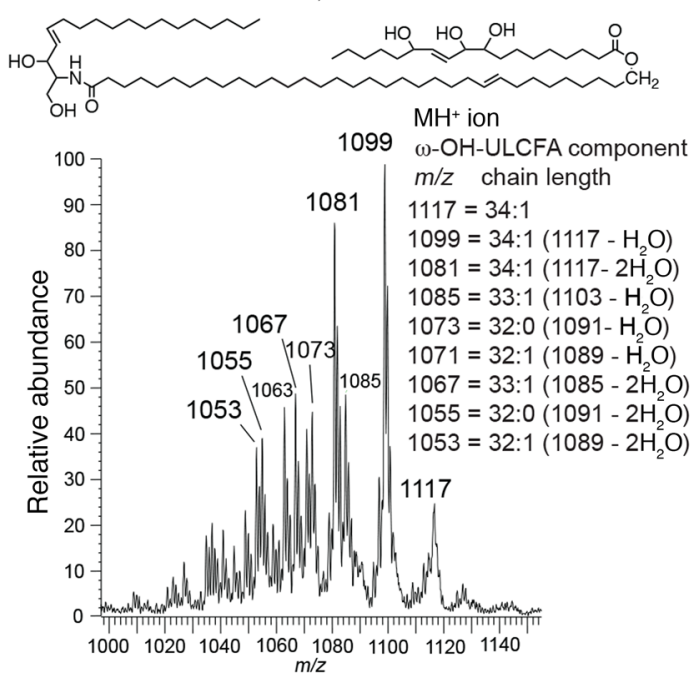

Figure 3. Epidermal accumulation of EOS-triol in Sdr9c7 null mice. (A) Representative TLC analysis of lipids extracted from $5 d r 9 c 7^{+/+}, S d r 9 c 7^{+/-}$, and $S d r 9 c 7^{-1-}$ epidermis. Sdr9c $7^{-1-}$ mice show the appearance of a major new polar lipid running between CerOS and Clc-CerEOS. In all $3 \mathrm{Sdr} g c 7 \mathrm{genotypes,}$ note the normal appearance of CerEOS, which is absent in the Pnpla1 knockout (right lane). The experiment was repeated 4 times with consistent results. (B) Normal-phase LC-MS analysis of WT and knockout $S d r 9 c 7$ epidermal lipids. The chromatograms show the total ion current profile ( $m / z$ 950-1350) of LC-MS analysis of $\mathrm{MeOH} / \mathrm{CHCl}_{3}$ epidermal extracts of WT (top panel, blue profile), and Sdr9c7-- (bottom panel, red profile) using an Advantage 5- $\mu \mathrm{m}$ silica column $(250 \times 4.6 \mathrm{~mm})$ run with gradient elution of hexane/isopropanol/glacial acetic acid (95:5:0.1) to hexane/isopropanol/glacial acetic acid (75:25:0.1) over 30 minutes using a Waters Alliance 2690 HPLC system coupled to a TSQ Vantage mass spectrometer. Note the prominent peak at approximately 20.5 minutes in the knockout, which is undetectable using this methodology in the WT. (C) Partial mass spectrum ( $m / z$ 1000-1150) of the 20.5-minute peak, with ion fragments identifying the product as CerEOS containing esterified trihydroxy-C18:1 fatty acid (EOS-triol).

bound CerOS from the protein pellets from $S d r 9 c 7^{+/+}$and $S d r 9 c 7^{-1-}$ skin samples revealed a $90 \%$ reduction of CerOS in the $S d r 9 c 7$ knockout epidermis (Figure 2G). Accordingly, we can conclude that the biochemical changes resulting from an absence of catalytically active SDR9C7 lead to a failure to produce covalently bound ceramides, with the resulting physiological defects in the skin barrier.

SDR9C7 is a dehydrogenase on epidermal lipoxygenase products. To establish the catalytic capabilities of SDR9C7, the recombinant human enzyme and the 2 naturally occurring mutants identified in ARCI patients were produced in insect cells and partially purified using FLAG-tagged affinity columns. The missense mutation identified in the new patient, R276C, gives the same sized protein band as WT on SDS-PAGE ( $\sim 33 \mathrm{kDa})$, and the nonsense mutation, $\mathrm{R} 220^{*}$, with a premature stop codon, results in an approximately 25 kDa protein band (Supplemental Figure 16, A-D). The amino acid sequence of SDR9C7 contains the characteristic residues for utilizing $\mathrm{NAD}^{+}$as cosubstrate (27), allowing use of the standard spectrophotometric assay for NADH production $(\lambda \max , 340 \mathrm{~nm})$ in enzyme assays. Catalytic activities were thus assessed for several lipoxygenase derivatives of linoleic acid as potential substrates, with subsequent HPLC identification of products by comparison to authentic standards (Figure 5A).

Using the NADH assay, followed by HPLC analysis of products, we identified oxidized linoleates of the $12 R$-LOX/eLOX3 pathway as SDR9C7 substrates. Methyl ester derivatives are metabolized by SDR9C7 at more than 10-fold higher rates than the free acids; efficient metabolism of the esterified substrates is significant because the natural linoleate substrates in the epidermis are esterified in CerEOS. Figure 5A illustrates the production of NADH in the course of oxidation of 13-hydroxy-linoleate methyl ester (13-HODE-Me) and epoxy-alcohol 9R,10R-transepoxy-11E-13R-hydroxy-octadecenoate methyl ester (RRR-EpOH$\mathrm{Me}$ ). Both the SDR9C7 R276C mutant (Figure 5A, bottom panel) and the $\mathrm{R} 22 \mathrm{O}^{*}$ mutant with premature stop codon were completely inactive, providing a biochemical correlate with their association with ARCI phenotype in vivo. Under our conditions, the rates of SDR9C7-catalyzed oxidation of 9-HODE-Me, 13-HODE-Me, $R R R-\mathrm{EpOH}-\mathrm{Me}$, and RSR-Triol-Me were similar, each attaining an increase in $340 \mathrm{~nm}$ absorbance of $\mathrm{NADH}$ of approximately $0.06 \mathrm{AU}$ in 10 minutes at room temperature, representing $10 \%$ conversion of the $1 \mathrm{mM} \mathrm{NAD}^{+}$cosubstrate to NADH. The SDR9C7 oxidation products were extracted and analyzed by HPLC-UV in comparison to authentic standards, thus establishing that the HODE-Me substrates are converted to the corresponding 9- or 13-ketone, and the RRR-EpOH-Me converted purely to the corresponding 13-ketone (Figure 5, B and C).

The chemical structure of prostaglandins includes a $13 E-15-$ hydroxyl moiety equivalent to the $11 E$-13-hydroxyl on the linoleate products. Furthermore, prostaglandins are metabolized in vivo by 15-hydroxyprostaglandin dehydrogenase, an SDR family member (SDR36C1) that converts the allylic 15-hydroxyl to a ketone. However, using a sensitive HPLC-UV assay, no activity was detectable with SDR9C7 and $\mathrm{PGE}_{2}$ or $\mathrm{PGE}_{2}$ methyl ester, indicating a distinct substrate specificity that can distinguish the similar $\omega 6$ 
A

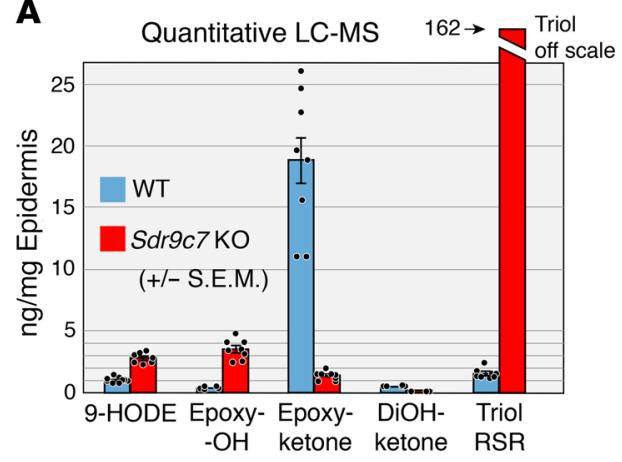

B

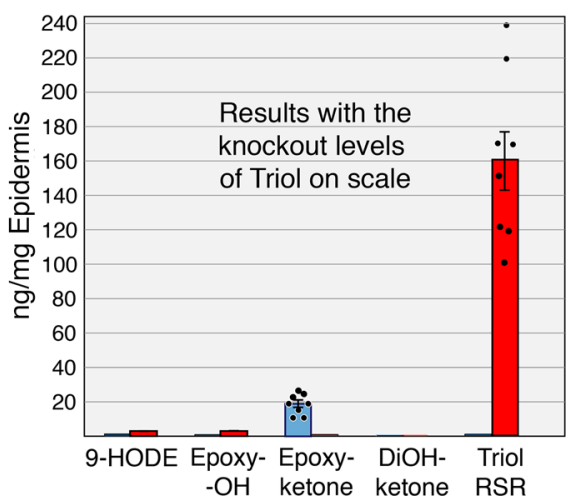

C

LC-MS of Triols
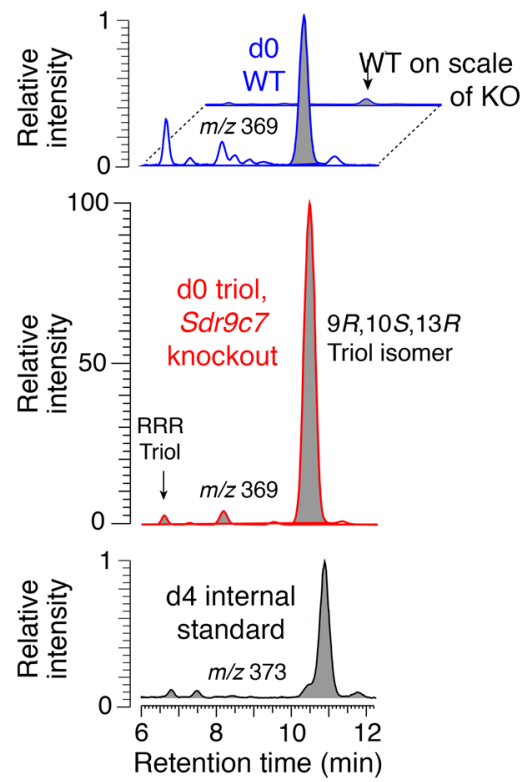

LC-MS of Epoxy-ketone
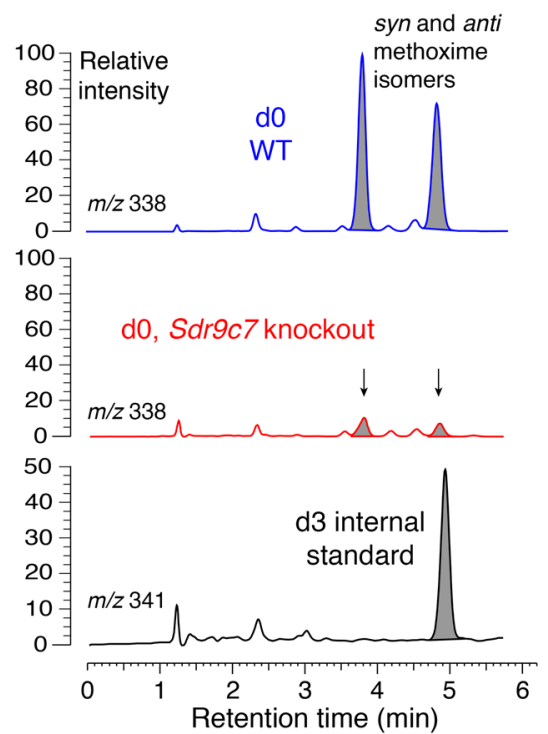

Figure 4. Quantitative LC-MS of oxidized linoleates esterified in WT and Sdr9c7 $7^{-1-}$ (Sdr9c7 KO) epidermis. (A) Quantitation of 9-HODE, 9,10-trans-11E-13hydroxy (Epoxy-OH), 9,10-trans-epoxy-11E-13-ketone (Epoxy-ketone), 9,10-dihydroxy-11E-13-ketone (DiOH-ketone), and 9R,10S,13R-trihydroxy-linoleate (Triol RSR). (B) The same data from A, shown on scale with the Sdr9c7 knockout levels of Triol RSR. Results are expressed in ng/mg epidermis (mean \pm SEM, $n=8$, $P<0.001$ for all pairs of WT compared with knockout, except for DiOH-ketone $[n=4, P<0.05]$ ). (C) LC-MS ion chromatograms illustrating analysis of linoleate

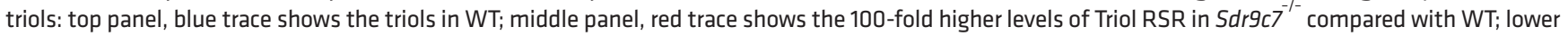
panel, black trace shows the $\mathrm{d} 4$ triol internal standard used for quantitation. (D) LC-MS ion chromatograms illustrating analysis of the Epoxy-ketone, with WT (top, blue trace), knockout (middle, red trace), and d3 epoxy-ketone internal standard (lower, black trace). Samples are analyzed with the 13-ketone derivatized to a 13-methoxime, which gives syn and anti isomers (the 2 peaks in the $\mathrm{d0}$ channel); the $\mathrm{d} 3$ internal standard was added as a single methoxime isomer, hence only one peak appears in the lower chromatogram. Oxidized lipids were isolated for LC-MS analysis as described in the Supplemental Data.

allylic hydroxyls of prostaglandins and the linoleate 12R-LOX/ eLOX3 metabolites. Finally, E. coli lysates of expressed SDR9C7 were reported to show weak NADH-dependent reduction of all-trans-retinal to all-trans-retinol (26). With our partially purified SDR9C7, there was no detectable $\mathrm{NAD}^{+}$or NADH-dependent inter-conversion of all-trans-retinal and all-trans-retinol, whereas side-by-side incubations with $R R R$-EpOH-Me showed more than $50 \%$ transformation to the 13-ketone.

\section{Discussion}

SDR9C7 is essential for epidermal barrier formation. Clinical observations reinforced by the phenotype of the knockout mouse confirm the physiological functioning of SDR9C7 as crucial for formation of the epidermal permeability barrier. Human patients show the typical ARCI features of dry skin, scaling, and hyperkeratosis. In our mouse knockout model, the skin of the $S d r 9 c 7^{-1-}$ pups failed to exclude toluidine dye and there was loss of body weight associated with TEWL. Analyses of the epidermis of both the patient and the knockout mouse revealed a severe depletion of the covalently bound ceramides, consistent with ultrastructural studies in the human patient showing failure to form the CLE. Significantly, the present mutation we identified in the patient (p.Arg276Cys) resulted not only in loss of catalytic activity, as we demonstrated with the recombinant enzyme, but also the mutation is associated with reduced expression in the stratum corneum and the stratum granulosum (Figure $1 \mathrm{C}$ ). This reduced expression was observed in some previous reports (29, 31 ), although it is not a universal feature of ARCI associated with SDR9C7 mutations (30).

The pathway of ceramide oxidations in the epidermis. Based on the LC-MS analysis of oxidized linoleates in the epidermal ceramides, together with the catalytic activities we identified for SDR9C7, we conclude that the oxidative pathways in forming the epidermal barrier proceed via LOX-catalyzed transformations of CerEOS followed by SDR9C7-catalyzed formation of an epoxy-enone derivative. As expanded on below, we propose that the chemical reactivity of the epoxy-enone with protein holds the key to the role of the enzymatic pathway in forming the CLE. Figure $6 \mathrm{~A}$ illustrates the normal oxidative pathway, and Figure $6 \mathrm{~B}$ is intended to convey a visual impression of the alterations when SDR9C7 is blocked or absent. As established by LC-MS analysis of the oxidized ceramides, loss of SDR9C7 leads to accumulation of the pathway precursors (CerEOS, CerEOS-9-HODE, CerEOSepoxy-alcohol) as well as a huge accumulation of CerEOS-triol as 
A
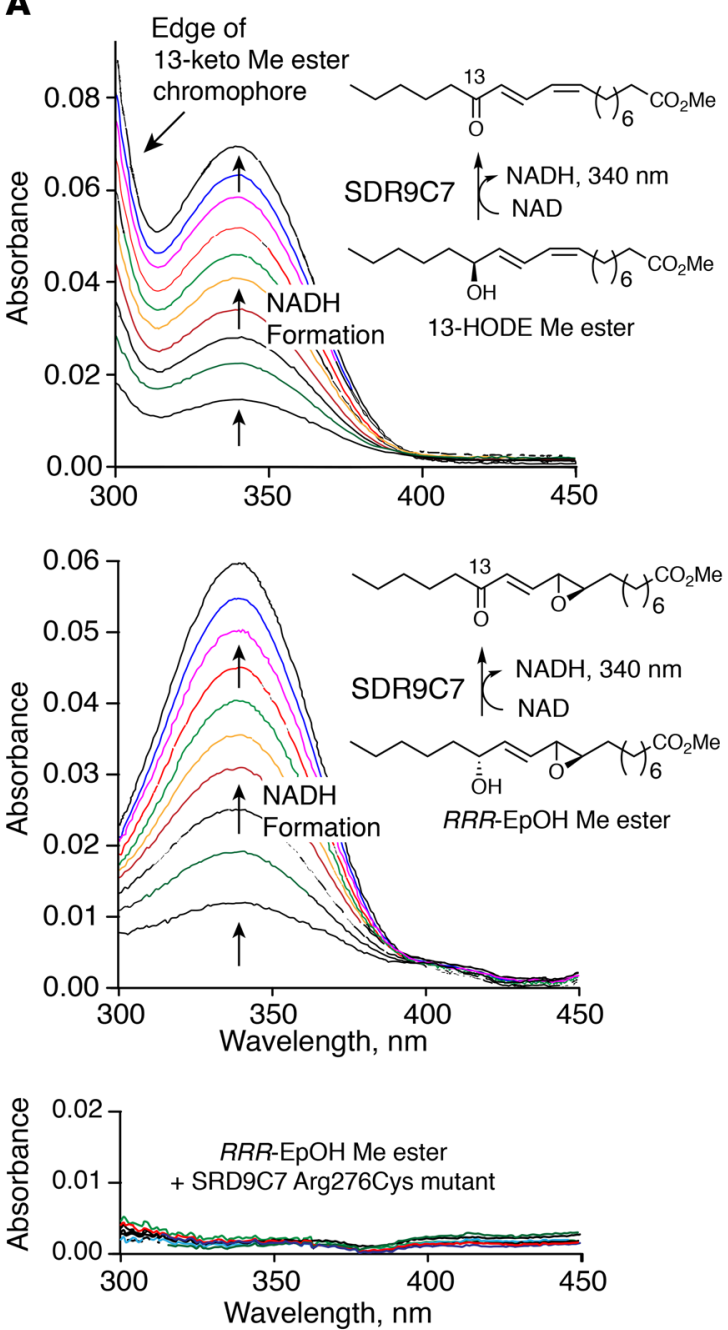

B

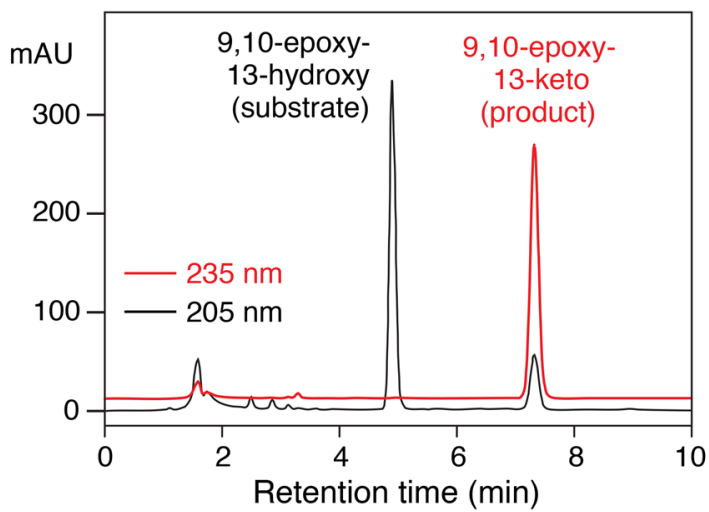

C

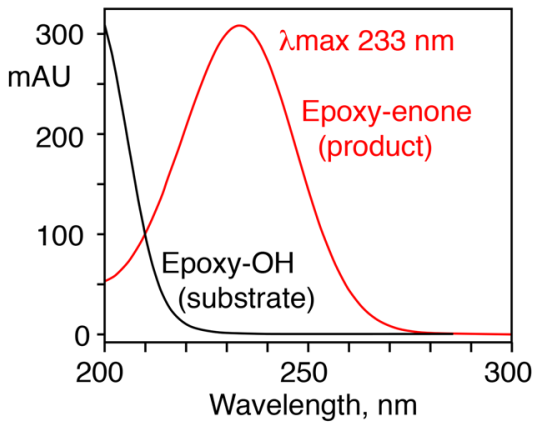

Figure 5. SDR9C7 catalyzes NAD+-dependent oxidation of 13-hydroxy-linoleate derivatives. (A) Activity of recombinant SDR9C7 monitored by spectrophotometric assay of NADH formation. Top panel: SDR9C7 catalyzes oxidation of 13-HODE methyl ester (13-HODE Me) to the 13-ketone. In addition to the appearance of NADH at $340 \mathrm{~nm}$, the scans in the metabolism of 13-HODE show an increase near $300 \mathrm{~nm}$, which represents the edge of the UV chromophore of the 13-keto oxidation product $(\lambda \max$ at $279 \mathrm{~nm})$; this increase in absorbance is not evident in metabolism of the epoxy-alcohol (middle panel), because its oxidation product absorbs much lower in the UV ( $\lambda \max 231 \mathrm{~nm})$ (10). Middle panel: SDR9C7 catalyzes oxidation of the epoxy-alcohol 9R,10R-trans-epoxy-11E-13R-hydroxy-octadecenoate methyl ester (RRR-EpOH-Me) to the 13-ketone. Lower panel: the SDR9C7 R276C mutant has no detectable activity in oxidation of $R R R-\mathrm{EpOH}-\mathrm{Me}$, (as further confirmed by HPLC analysis of the incubation). (B) Reversed-phase HPLC analysis of the SDR9C7-catalyzed oxidation of RRR-EpOH-Me. The unreacted substrate is detected in the channel monitoring $205 \mathrm{~nm}$, the single product mainly at 235 $\mathrm{nm}$. (C) The 9,10-epoxy-11E-13-keto product was identified from its cochromatography with the authentic standard and by its characteristic UV spectrum. The HPLC analysis used an Agilent 1200 series system with an Agilent Eclipse XBD-C18 column ( $5 \mu \mathrm{m}, 15 \times 0.46 \mathrm{~cm})$, a solvent of acetonitrile/water/glacial acetic acid (75/25/0.01 by volume) at a flow rate of $1 \mathrm{~mL} / \mathrm{min}$, with the diode array detector set to monitor at 205, 220, 235, and 270 $\mathrm{nm}$.

a bypass route when $\mathrm{SDR} 9 \mathrm{C} 7$ is blocked. The normal route involving SDR9C7 forms CerEOS-epoxy-enone.

A new hypothesis for covalent binding via the chemical reactivity of CerEOS-epoxy-enone. We propose that the ultimate chemical or biochemical role of the SDR9C7-mediated transformation to CerEOS-epoxy-enone is to facilitate ceramide binding to the proteins of the CCE and thus initiate formation of the CLE (Figure $6 \mathrm{C})$. In terms of the chemical reactivity of the epoxy-enone moiety created by SDR9C7, there is ample literature precedent to support a role in protein binding. A plain $\alpha / \beta$-unsaturated ketone (simply an enone) is reactive via Michael addition to cysteine, histidine, and lysine amino acid residues (Figure 6D) $(2,36,37)$. Furthermore, reaction with amino groups will link the fatty acid to protein as a
Schiff base (Figure 6E) $(2,36,37)$. Although both such transformations are reversible, they could promote a close association of ceramide and protein for subsequent irreversible binding. In fact, an earlier study of LC-MS analyses of mouse epidermis detected the reversible protein binding of CerEOS-epoxy-enone (10). Addition of the epoxide group in conjugation with the enone moiety (epoxyenone) greatly enhances the reactivity with protein. Indeed, the very linoleate 9,10-epoxy-11E-13-keto-octadecenoate as formed through the LOX/SDR9C7 pathway is a well-established model compound used to define the chemistry of lipid-protein binding $(38,39)$. It is shown to form pyrrole and furan derivatives in irreversible covalent reaction with lysine and other protein residues (Figure 6E). Thus, we envisage the key role for SDR9C7 in forming 


\section{A}

Normal pathways, normal levels of products

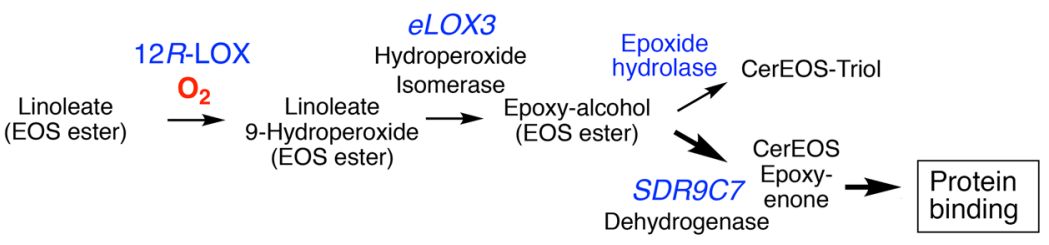

\section{B}

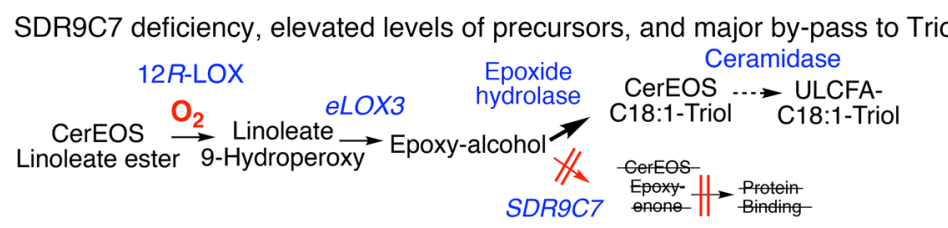

C

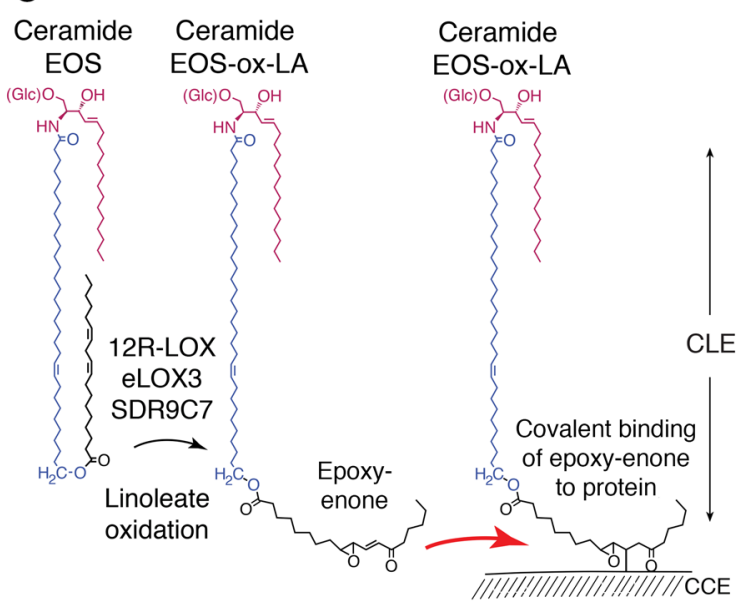

D Michael addition reactions

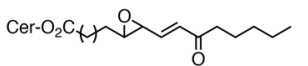

$$
+\mathrm{R}-\mathrm{SH} \downarrow \text { Cysteine }
$$

Cer- $\mathrm{O}_{2} \mathrm{C}_{4}+\mathrm{R}_{\mathrm{R}-\mathrm{S}}^{\mathrm{O}}$

Michael adduct with cysteine

\section{E}

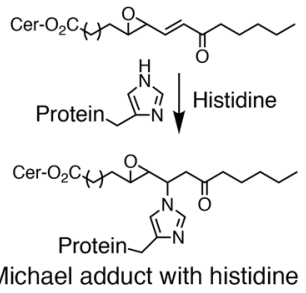

Schiff base and pyrrole formation

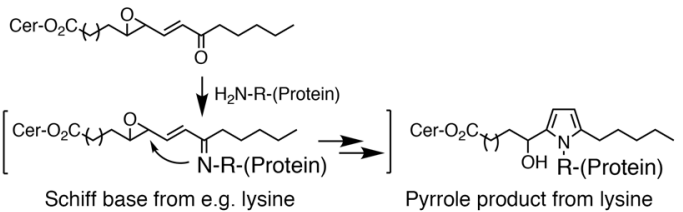

Figure 6. Potential modes of protein binding by covalent reactions of EOS-epoxy-enone. (A) The normal pathways of oxidation of linoleate esterified in CerEOS produces a reactive epoxy-enone that can bind to protein. (B) SDR9C7 deficiency almost abolishes protein binding and formation of the CLE and induces a major bypass shunt pathway in metabolism toward the CerEOS-triol. (C) Conversion of CerEOS via the actions of 12R-LOX, eLOX3, and SDR9C7 creates a reactive epoxy-enone moiety capable of reacting nonenzymatically to form covalent bonds with CCE proteins, thus forming the CLE. (D) Michael addition reactions of the enone moiety form reversible bonds with Cys or His residues. (E) Reaction of the epoxy-enone moiety with free amino groups forms a Schiff base that can rearrange to form irreversible bonding via pyrrole formation.

CerEOS-epoxy-enone for covalent reaction with protein and leading to formation of the CLE (Figure 7).

Points in favor of the new hypothesis. For the last several years, a credible hypothesis on the functioning of the lipoxygenase pathway has foreseen a role for the LOX products including EOStriol, based on their high polarity. This was considered to facilitate esterase-catalyzed cleavage of the oxidized linoleate, thus providing free CerOS as a substrate for covalent coupling to protein (10, 13, 34). However, the massive accumulation of CerEOS-triol in the $S d r 9 c 7$ knockout animals suggests that an esterase-catalyzed cleavage of the linoleate-triol from the ceramide is not the functional pathway. If it were, the release of nonesterified linoleatetriol would be predicted, whereas in fact, judging from the huge accumulation of CerEOS-triol, it appears that ester-catalyzed release of free triol is not operative.

This conclusion concurs with the very low levels of free linoleate triol measured in normal human and murine epidermis (34), albeit modestly elevated levels $(<0.2 \mathrm{pg} / \mu \mathrm{g}$ protein) are reported in association with atopic dermatitis and are suggested as a biomarker of barrier function (8). According to our new concept, there is no requirement for the unidentified missing esterase (5, 13) to remove the oxidized linoleate and provide free CerOS for coupling to protein. Also, for this proposed early stage of forming the covalently bound ceramides, the enzyme transglutaminase is not required for covalent binding.

Slightly as an aside, although of indirect relevance, there are several ARCI genes whose inactivation leads to accumulation of CerOs, the ceramide with the free omega-hydroxyl that can bind to protein glutamate residues via ester linkage $(17,40)$. Yet in the case of inactivation of PNPLA1 or CGI-58 (ABHD5) $(22,24,41)$, a prime structural defect in the barrier is loss of the CLE. One might reasonably question why there is accumulation of what is generally considered to be the main substrate for covalent binding (CerOS), yet formation of the CLE is impaired? Possibly this could be explained by the direct binding of CerOS not being the major pathway involved in initiation of covalent protein binding.

A further concept stemming from the huge accumulation of CerEOS-triol in the $S d r 9 c 7$ knockout is that normally there is a sub- 


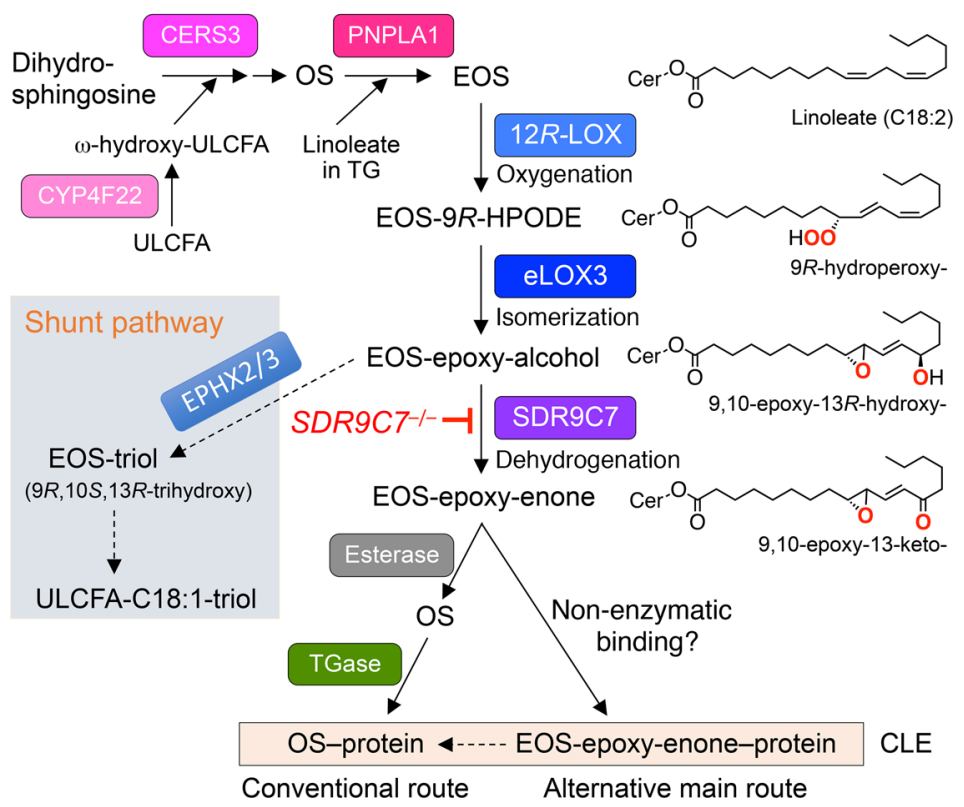

Figure 7. Role of SDR9C7 in the routes of ceramide oxidation leading to covalent binding to protein and formation of the CLE. Top: CerEOS is synthesized via $\omega$-hydroxylation of ULCFA by CYP4F22, the coupling of $\omega$-hydroxy-ULCFA with dihydrosphingosine by CERS3 and subsequent desaturation to form CerOS, and transacylation of linoleate from triglyceride (TG) to CerOS by PNPLA1. Middle: The linoleate in CerEOS is oxygenated by $12 R-$ LOX to the $9 R$-hydroperoxide, isomerized by eLOX3 to the RRR-epoxy-alcohol, followed by dehydrogenation by SDR9C7 to form the reactive CerEOS-epoxy-enone. Last steps: Covalent coupling to protein may occur by the conventionally understood mechanism involving hydrolysis of the oxidized linoleate and binding of the resulting CerOS to protein via uncharacterized esterase and transglutaminase (TGase) enzymes (left-hand route), and/or by direct binding of CerEOS-epoxy-enone to CCE proteins thus forming covalent bonds, potentially via nonenzymatic reactions of the epoxy-enone moiety (right-hand route). stantial flux through the pathway in the WT epidermis. It may be that in the normal epidermis the route to CerEOS-triol serves as a finetuning valve in regulating the production of CerEOS-epoxy-enone.

Assessing the evidence for ceramide binding in the CLE via ester bonds to glutamate. In a classic and well-cited paper by Marekov and Steinert (40), the corneocyte envelopes from human foreskin tissue were purified, proteolytically digested, and the resulting lipidpeptide fragments arising from the covalently bound CLE were analyzed by LC-MS. In the lipopeptides isolated, the lipid components were comprised mainly of ceramides of 690 to 890 molecular mass; they were bound to a glutamate residue in identified peptide fragments from the CLE protein involucrin. The molecular mass of the lipid component is within the correct range to include CerOS, and too light in mass to be CerEOS (of mass range 950-1100 when linoleate or oxidized linoleate is the ester-linked fatty acid). In support of the findings, Nemes and Steinert showed that, in vitro, transglutaminase can form an ester linkage between a CerOS analogue and glutamate residues in involucrin protein (17). Together, the 2 studies represent impressive technical accomplishments and establish that the direct linkage of CerOS to protein via an ester bond to glutamate is at least a significant component of the CLE.

To the best of our knowledge, the Marekov and Steinert paper (40) is the only study reporting directly on modes of ceramide-protein linkage in the epidermis. To what extent does this pioneering work leave open the possibilities for additional modes of lipoprotein interaction and covalent binding? For a start, the identified lipoprotein fragments accounted for about $20 \%$ for the total estimated ceramide binding to the human foreskin CCE (40), leaving space for additional modes and mechanisms. Second, involucrin, the primary CLE protein involved in the identified lipopeptides, is not itself essential for barrier function (there is considerable redundancy in regard to CCE proteins) $(42,43)$, suggesting the measured lipopeptides do not in themselves represent vital components of the barrier. Third, all further studies on the topic of covalent binding of ceramide to the CCE used mild alkaline hydrolysis to release the free ceramide, an approach that cannot distinguish between the release of free CerOS from ester bonding to glutamate (as universally interpreted), to the release of the same free CerOS from bonding induced via covalent reaction with CerEOS-epoxy-enone (Figure 6).

Summing up. In the early stages of construction of the CCE and covalent binding of ceramide, nucleation of the incipient structures is focused around desmosomes (44), and it is at this stage that we foresee a critical role for covalent reaction of CerEOS-epoxy-enone to initiate lipoprotein bonding. Indeed, synthesis of the epoxy-enone moiety itself must occur in viable cells with availability of $\mathrm{NAD}^{+}$cofactor, a surmise that matches with the recent conclusion that $12 R$-LOX and eLOX3 create the oxygenated CerEOS in viable cells at the relatively early developmental stages of creation of the barrier (16). The reactivity of the epoxy-enone moiety can initiate binding via Michael addition, and this could become irreversible through pyrrole formation as established over 2 decades ago in model studies using the methyl ester of linoleate 9,10-epoxy-11trans-13-ketone $(38,39)$. While it will be a considerable technical challenge to detect these formative structures, our work identifies specific possibilities and sets new goals for future barrier-related investigations.

\section{Methods}

Generation of the Sdr9c7 knockout mice. C57BL/6J mice were purchased from Japan SLC. All mice were fed a commercial CE-2 diet (CREA Japan) and had ad libitum access to water. The mice were bred in a pathogen-free facility at the Institute for Laboratory Animal Research, Graduate School of Medicine, Nagoya University (45), and maintained under a controlled temperature of $23^{\circ} \mathrm{C} \pm 1^{\circ} \mathrm{C}$, humidity of $55 \% \pm 10 \%$, and a light cycle of 12 -hours light (from 09:00 to 21:00)/12-hours dark (from 21:00 to 09:00). Animal care and all experimental procedures were approved by the Animal Experiment Committee, Graduate School of Medicine, Nagoya University, and were conducted according to the Regulations on Animal Experiments of Nagoya University. 
Targeted disruption of the $S d r 9 c 7$ gene on a C57BL/6J background was carried out by using the CRISPR/Cas9 method as previously described (46). The CRISPR RNA (crRNA, 5 '-CAACCAGTTGTTTGGCCAAC-3') that targets exon 1 was designed using the CRISPOR website (47). The designed crRNA and trans-activating crRNA (tracrRNA) (Genome CraftType CT, FASMAC) and Cas9 protein (New England Biolabs) were mixed and incubated at $37^{\circ} \mathrm{C}$ for 20 minutes to form a ribonucleoprotein complex (RNP). We initially hypothesized that pathogenic biallelic mutations in $S d r 9 c 7$ in mice might result in embryonic lethality. Therefore, we mixed the single-stranded donor oligonucleotide (ssODN) with RNP preparation to generate founder mice carrying heterozygous knockout alleles of the $S d r 9 c 7$ gene. The ssODN (5'-TCTTCATCACTGGCTGTGACTCCGGCTTTGGGAATCTGTTGGCCAAACAACTGGTTGATAGGGGCATGAAAGTGCTTGCT-3') was designed to include a silent (synonymous) c.117C $>$ T mutation to avoid cleavage by Cas 9 and was obtained from FASMAC. The final concentrations of components in RNP preparation with ssODN were $8 \mu \mathrm{M}$ guide RNA (crRNA + tracrRNA), $200 \mathrm{ng} / \mu \mathrm{L}$ Cas 9 protein, and $250 \mathrm{ng} / \mu \mathrm{L}$ ssODN. The mixture was electroporated into zygotes using a NEPA 21 electroporator (NEPA GENE Co. Ltd.) and the embryos were transferred into the oviductal ampulla of pseudopregnant Institute of Cancer Research mice.

For sequencing and genotyping, genomic DNAs were extracted using KAPA Express Extract (Kapa Biosystems) from the pinna and tail of the offspring and used for PCR amplification. The target region by the Cas 9 nuclease was amplified by using a GoTaq Green Master mix (Promega) and a primer pair (5'-CTGTTGCAAGTCTCAGAAGCTCTC-3' and 5'-GACATCTAGGAGGAAGGTCTGCAG-3'). Mutations in the $S d r 9 c 7$ gene in offspring were confirmed by Sanger sequencing of the PCR products using Eurofins DNA sequence service (Eurofins Genomics) (Supplemental Figure 2B). Potential off-target cleavage sites predicted by the CRISPOR website (Supplemental Table 10, top 5 high-scored regions according to the Mit off-target score) were sequenced and no mutations were detected in these sites.

Immunohistochemistry. Immunohistochemical analysis of skin samples from the participants and mice was performed as described previously (48), with slight modifications. Thin sections $(3 \mu \mathrm{m})$ were cut from samples embedded in paraffin blocks. The sections were soaked for 20 minutes at room temperature in $0.3 \% \mathrm{H}_{2} \mathrm{O}_{2} /$ methanol to block endogenous peroxidase activity. After washing in PBS with $0.01 \%$ Triton $\mathrm{X}-100$, the sections were incubated for 30 minutes in PBS with $4 \%$ BSA, followed by an overnight incubation with the primary antibodies in PBS containing 1\% BSA. After washing in PBS, the thin sections were stained with biotinylated goat anti-rabbit immunoglobulin secondary antibodies for 1 hour at room temperature and washed in PBS. The Vectastain Elite ABC-PO kit (Vector Laboratories) was used for staining. The polyclonal antibody for anti-SDR-O was purchased from Abcam (ab90371).

TLC. After subcutaneous tissue was removed by scraping on ice, skin pieces were incubated in $\mathrm{PBS}$ at $60^{\circ} \mathrm{C}$ for 1 minute, and the epidermis was peeled from the dermis. The isolated epidermis was vortex-homogenized with steel beads in $1 \mathrm{~mL}$ methanol using bead crusher $\mu$ T-01 (TITEC). Free lipids were extracted by a modified Folch method. Epidermal lipids corresponding to $5 \mathrm{mg}$ dry weight were separated by TLC (Silica gel 60, Merck) with the following solvent sequence: chloroform/methanol/water (40:10:1) to $2 \mathrm{~cm}$; chloroform/ methanol/water (40:10:1) to $5 \mathrm{~cm}$ : chloroform/methanol/acetic acid
(47:2:0.5) to $8 \mathrm{~cm}$; $n$-hexane/diethyl ether/acetic acid (65:35:1) to the top of the plate. Lipids were visualized by spraying the plate with $5 \%$ (wt/vol) $\mathrm{CuSO}_{4}$ in $15 \%$ (vol/vol) $\mathrm{H}_{3} \mathrm{PO}_{4}$ followed by heating at $180^{\circ} \mathrm{C}$ for 15 minutes. Lipid classifications were performed by comparison with authentic lipid standards.

Preparation of oxidized linoleates and deuterated internal standards. Hydroxy-octadecadienoates (HODEs), linoleate epoxy-alcohols, and linoleate triols were prepared as described $(49,50)$. The corresponding 13-ketones of the epoxy-alcohol and triol were prepared as described in detail in Supplemental Methods. All intermediates and final products were purified and characterized by HPLC, UV, and ${ }^{1} \mathrm{H}-\mathrm{NMR}$. The deuterated internal standards of 9-HODE, 9R,10R-transepoxy-11E-13R-hydroxyoctadecenoate, and the $9 R, 10 S, 13 R$-triol for LC-MS quantitative assays were prepared from $\left[9,10,12,13-{ }^{2} \mathrm{H}_{4}\right]$ linoleic acid as described (34). The deuterated internal standards for assay of the corresponding 13-ketones of the epoxy-alcohol and triol were prepared by derivatization of the free acid with trideuterated methoxylamine hydrochloride $\mathrm{CD}_{3} \mathrm{ONH}_{2} \cdot \mathrm{HCl}$ (CDN Isotopes).

Quantitative LC-MS analysis of oxidized linoleate-containing ceramides in murine epidermis. The epidermis of neonatal WT and $S d r 9 c 7^{-1-}$ pups was extracted with $\mathrm{MeOH} / \mathrm{CHCl}_{3}$ and the lipids processed with minor changes from previous methods (34), as described in detail in Supplemental Methods. Briefly, after 3 washes with $\mathrm{MeOH} / \mathrm{CHCl}_{3}$, the pooled lipid extract was chromatographed on an open-bed silica cartridge and the ceramide fraction subsequently treated with methoxylamine hydrochloride in pyridine to convert fatty acid keto groups to the methyloxime derivative. Deuterated internal standards (0.1 nmol, $30 \mathrm{ng}$ ) were then added for analysis of 9-HODE, 9R,10R-trans-epoxy-11E-13-hydroxy-octadecenoate, $9 R, 10 R$-trans-epoxy-11E-13-keto-octadecenoate, $9 R, 10 S$-dihydroxy-11E-13-keto-octadecenoate, and 9R,10S,13R-trihydroxy-11E-octadecenoate. Alkaline hydrolysis of the esterified lipids was followed by recovery of the oxidized linoleates using a reversed-phase Oasis cartridge (Waters), formation of the pentafluorobenzyl esters, and for analysis of the dihydroxy-13-keto- and trihydroxy-linoleates, conversion to the 9,10-dimethoxypropyl (DMP) acetonide derivative, as described previously in detail (34).

The PFB or PFB-DMP derivatives were analyzed as the M-PFB ions by APCI-LC-MS using a TSQVantage instrument (Thermo Fisher Scientific) with the APCI vaporizer temperature set to $500^{\circ} \mathrm{C}$, and the capillary temperature set to $150^{\circ} \mathrm{C}$. The HPLC used a Waters Alliance 2690 system and a Phenomenex Luna $5 \mu \mathrm{m}$ silica column $(25 \times 0.2 \mathrm{~cm})$ with a flow rate of $0.6 \mathrm{~mL} / \mathrm{min}$, and a solvent of hexane/isopropanol in the proportions 100:0.2 (vol/vol) for analysis of 9-HODE (recorded at $m / z 295$ [d0] and $m / z 299$ [d4]), 9,10-epoxy-13-hydroxy-C18:1 ( $\mathrm{m} / z$ 311 and $m / z$ 315), 9,10-epoxy-13-methoxime-C18:1 $(\mathrm{m} / z 338$ and $\mathrm{m} / z$ 341 [d3]), 9,10-dihydroxy DMP 13-methoxime $(\mathrm{m} / z 396$ and $\mathrm{m} / z$ 399) and the proportions 100:1 (vol/vol) for analysis of linoleate triols (PFB, DMP derivative recorded at $m / z 369$ [do] and $m / z 373$ [d4]).

Quantitation of CerOS covalently bound to epidermal proteins was carried out essentially as previously described (10), as detailed in Supplemental Methods. The LC-MS analyses used an LTQ linear ion trap mass spectrometer (Thermo-Electron) equipped with an Ion Max APCI source and recording positive ions $(m / z 700-900)$ in full scan mode, and a Phenomenex Luna $5 \mu \mathrm{m}$ silica column with a solvent of hexane/isopropanol/glacial acetic acid, 90:10:0.1 (vol/vol/vol) at a flow rate of $0.6 \mathrm{~mL} / \mathrm{min}$, with retention time for CerOS of approximately 4 minutes. 
A patient with lamellar ichthyosis. The patient was a 22-year-old Japanese woman, the fourth daughter born to nonrelated parents without any family history of similar disorders (Supplemental Figure 1D). At birth, she showed a collodion membrane on her entire body surface. Then, she presented with generalized ichthyosis. Examination revealed diffuse brown shiny scales on the trunk and extremities. She also had mild palmoplantar keratoderma (Figure 1A, Supplemental Figure 1, A and B). Her skin tends to be milder when she is in a high temperature and humid environment. Her hair, nails, and teeth were normal. She did not have any neurological symptoms or hearing loss. Her serum biochemistry showed the normal vitamin A level, $530 \mathrm{ng} /$ $\mathrm{mL}$ (normal range, 431-1041 ng/mL).

Analysis of the patient's stratum corneum lipid by tape stripping and LC-MS. To examine the ceramide species present in the stratum corneum, tape stripping was performed by pressing and stripping an adhesive acrylic film (465 no. 40; Teraoka Seisakusho) from the skin of the right upper arm and forearm of the patient and her parents. Samples were also taken from the same lesions of 8 normal controls (healthy women in their twenties or thirties) as controls. Five strips measuring $25 \times 50 \mathrm{~mm}$ each were obtained from a single individual. Half of the samples were then subjected to LC-MS analysis to assess the levels of 11 major ceramide species (51-53) and 2 bound ceramide species.

The strips were cut into half-strips, $25 \times 15 \mathrm{~mm}$ in size: one for LC-MS analysis and the other for protein analysis. The lipids within the first half-strip were dissolved in $2 \mathrm{~mL}$ chloroform/methanol/2-propanol (volume ratio: 10:45:45). $N$-heptadecanoyl- $D$-erythro-sphingosine (d18:1/17:0) (Avanti Polar Lipids) was added as an internal control, and its final concentration was $50 \mathrm{nmol} / \mathrm{L}$. We confirmed the amount of endogenous d18:1/17:0 ceramide (NS_C35) in the stratum corneum (Supplemental Table 12). The results show that a small amount of endogenous d18:0/17:0 ceramide is present in the stratum corneum and, estimated from the peak area, the amounts of NS_C33, NS_C34, NS_C35, NS_C36, and NS_C37 are almost the same.

The amount of endogenous d18:1/17:0 ceramide against the added synthetic standard is about $0.7 \%$. The ratio was calculated by the following formula: (peak area of the synthetic standard-free sample, 676) / (peak area of the synthetic standard-added sample, 98,023) $\times 100=0.69$.

We quantified all ceramide species by calculating the relative sensitivity of chain lengths and types from an approximate expression. This lipid solution was subjected to reverse-phase LC-MS. The system was an Agilent 6130 Series LC-MSD system equipped with a multi-ion source, ChemStation software, an Agilent 1260 Infinity Series LC (Agilent Technologies), and an L-column octadecylsilyl $(2.1 \mathrm{~mm}$ internal diameter $\times 150 \mathrm{~mm}$; Chemicals Evaluation and Research Institute). Chromatographic separation of the lipids was achieved at a flow rate of $0.2 \mathrm{~mL} /$ minute using a mobile phase of binary gradient solvent system. Each ceramide species was detected by selected ion monitoring of $m / z$ [M+acetate $]^{-}$and HPLC retention times specific to ceramide species (Supplemental Table 13). In detail, phospholipids, sphingolipids, and fatty acids are detectable in the negative ion mode, although phospholipids are almost not included in the sebum and the stratum corneum intercellular lipids. The other lipids in the skin, such as triglycerides, wax esters, cholesterol esters, and squalene, are not detected in the negative ion mode. Ceramides and fatty acids can be completely separated according to their mass and polarity differences. Some groups of ceramide species (NH, AS, and NDS; AP, ADS, and AH; EODS and EOH) may have identical masses depending on the specific combinations of sphingolipids and fatty acids. However, they can be discriminated by their specific retention times of HPLC, according to the polarity differences (Supplemental Table 13). Soluble proteins were extracted from the other half-strip with a $0.1 \mathrm{~mol} / \mathrm{L} \mathrm{NaOH}-1 \%$ sodium dodecyl sulfate solution at $60^{\circ} \mathrm{C}$ for 150 minutes, followed by neutralization with $\mathrm{HCl}$. The concentration of soluble proteins was determined using a BCA protein assay kit (Thermo Fisher Scientific).

Analysis of covalently bound ceramides in the stratum corneum. Five tape strips measuring $25 \times 20 \mathrm{~mm}$ were used for thin-layer chromatography according to the previously reported method (15) with a slight modification. Stratum corneum and tape adhesive components were removed from tape strips by suspending in $n$-hexane. Stratum corneum was collected by filtration with Durapore filter (Merck Millipore). The free lipids were removed from stratum corneum with the filter by suspending successively in 3 separate mixtures of chloroform/methanol (volume ratios: 2:1, 1:1, and 1:2) for 2 hours each. The last mixture (1:2) was filtrated with the PTFE filter (ADVANTEC) and the weight of dried stratum corneum collected by filtration was measured as difference of the filter weight. The covalently bound lipids were released from the remaining tissue on filter by mild alkaline hydrolysis with $1 \mathrm{~N}$ $\mathrm{KOH}$ in $95 \%$ methanol $60^{\circ} \mathrm{C}$ for 2 hours. After neutralization with $\mathrm{HCl}$, lipids were extracted with chloroform. The lipid extracts equivalent to $0.1 \mathrm{mg}$ dry weight of stratum corneum were applied onto HPTLC silica gel plate (silica gel 60, $0.2 \mathrm{~mm}, 10 \mathrm{~cm} \times 20 \mathrm{~cm}, 5065-32001$ ) (Merk Millipore), and developed twice with chloroform/methanol/ acetic acid (volume ratio: 190:9:1, to the top). After chromatography, the plates were sprayed with $10 \% \mathrm{CuSO}_{4} / 8 \% \mathrm{H}_{3} \mathrm{PO}_{4}$ aqueous solution and charred on a hot plate at $180^{\circ} \mathrm{C}$ for 5 minutes. The distribution of components was determined by scanning densitometry with EPSON SCAN (GT-X820, CS Analyzer) (Supplemental Figure 1E). The quantification of covalently bound ceramides was performed by comparison with commercially available ceramide standards run on the same plates (ceramides [nonhydroxy] and ceramides [hydroxy]) (Matreya).

Expression, purification, and assay of human and mouse SDR9C7. cDNA of full-length human SDR9C7 was purchased from Kazusa Genome Technologies. The substitution c.826C $>\mathrm{T}$ (p.Arg276Cys) is a mutation in the ARCI patient of the present study, whereas the truncation c.658C $>\mathrm{T}\left(\mathrm{p} . \operatorname{Arg} 220^{*}\right)$ was a previously reported mutation in the patient with ARCI from another group (30). Human SDR9C7 proteins (WT, R276C and R220*) were expressed including an N-terminal FLAG tag in a silkworm-baculovirus system, ProCube (Sysmex Corporation) as described elsewhere (54). Expression constructs of the mouse Sdr9c7 were synthesized by BioBasic, transferred into pET28b vector, and expressed in E. coli. This produced strong enzymatic activity in the bacterial lysate $(100 \mu \mathrm{L}$ lysate/mL completely metabolized $100 \mu \mathrm{M} 13-\mathrm{HODE}$ free acid in 10 minutes at $37^{\circ} \mathrm{C}$ ); however, despite testing $\mathrm{N}$-terminal and $\mathrm{C}$-terminal $\mathrm{His}_{6}$ tags in the constructs, the activity was not captured on a nickel affinity column. Because of ester hydrolase activity in the crude lysate, these preparations were of limited utility for analysis of esterified substrates.

Enzyme incubations with FLAG tag-purified SDR9C7 were conducted at room temperature in a total volume of $0.5 \mathrm{~mL}$ in $0.1 \mathrm{M}$ sodium phosphate $\mathrm{pH} 7.4$ containing $1 \mathrm{mM} \mathrm{NAD}^{+}, 100 \mu \mathrm{M}$ substrate (e.g., epoxy-hydroxy free fatty acid or methyl ester), $0.1 \%$ polyoxyethylene tridecyl ether detergent (MilliporeSigma), and 1-2 $\mu \mathrm{g}$ enzyme. Rates of reaction were monitored by UV spectroscopy for appearance of 
the NADH chromophore ( $\lambda \max 340 \mathrm{~nm}$, recorded for 10 minutes by repetitive scans at 1-minute intervals over the range $300-450 \mathrm{~nm}$ ).

After continuing the incubation for 30 minutes at room temperature, enzymatic products were extracted with dichloromethane or using a $30 \mathrm{mg}$ Oasis cartridge (Waters) with final elution using ethyl acetate or methanol. Initial screening of the products was conducted on an Agilent 1200 series HPLC system with the diode array detector set to monitor 205, 220, 235, and $270 \mathrm{~nm}$. Reversed-phase HPLC used a Waters Symmetry C18 column $(25 \times 0.46 \mathrm{~cm})$ with isocratic solvent systems of acetonitrile/water/glacial acetic acid adjusted from 80:20:0.01 ( $\mathrm{vol} / \mathrm{vol} / \mathrm{vol}$ ) to higher percentages of water depending on the polarity of the analytes, with a flow rate of $1 \mathrm{~mL} / \mathrm{min}$. Normal-phase HPLC used a Thompson Advantage silica column $(25 \times 0.46 \mathrm{~cm})$ with solvent systems of hexane containing $0.5 \%-5 \%$ isopropanol, depending on the polarity of the analytes. Chiral HPLC used a Chiralpak AD column $(15 \times 0.2 \mathrm{~cm}$ or $25 \times 0.46 \mathrm{~cm})$ with a solvent system of hexane/ EtOH/MeOH for epoxy-hydroxy methyl ester derivatives (10).

Data availability. The microarray data can be accessed at the GEO repository under the accession numbers GSE87682 (22) and GSE135643.

Statistics. Significance was determined by unpaired 2-tailed Student's $t$ test for normally distributed samples and using the nonparametric Mann-Whitney $U$ test (2-tailed) for quantitative LC-MS analyses. A $P$ value less than 0.05 was considered significant. All the data are mean \pm SEM (error bar).

Study approval. This study was approved by the ethics committee of the Nagoya University Graduate School of Medicine. All studies were conducted according to the Declaration of Helsinki Principles. Animal care and all experimental procedures were approved by the Animal Experiment Committee, Graduate School of Medicine, Nagoya University, and were conducted according to the Regulations on Animal Experiments of Nagoya University.

\section{Author contributions}

TT, TH, ARB, and MA designed the research studies. TT, TH, Y Miyasaka, AK, YO, K Tanahashi, HT, K Tanaka, MWC, WEB, JI,
TO, ARB, and MA conducted experiments. TT, TH, AK, YO, and $\mathrm{K}$ Tanahashi acquired data. TT, TH, AK, YO, K Tanahashi, JI, ARB, and MA analyzed data. TT, ST, and CM collected clinical samples and information. TT, TH, ARB, and MA wrote the manuscript. DW, MK, and Y Muro helped write the paper.

\section{Acknowledgments}

We thank the staff at the Division of Experimental Animals, Nagoya University Graduate School of Medicine, for their technical support. We also thank Yasumasa Nishito, Haruka Ozeki, Mitsuko Kobayashi, and Yuka Terashita for their valuable assistance. This work was supported by funding from Advanced Research and Development Programs for Medical Innovation (AMED-CREST) (19gm0910002h0105 to MA and $19 \mathrm{gm0910002h0205}$ to TH) from the Japan Agency for Medical Research and Development (AMED). This work was also supported by a Grant-in-Aid for Scientific Research (B) (18H02832 to MA) and by a Grant-in-Aid for Young Scientists (18K16058 to TT) from the Japan Society for the Promotion of Science (JSPS). This investigation was supported in part by The Mochida Memorial Foundation for Medical and Pharmaceutical Research, The Uehara Memorial Foundation, and The Kanae Foundation for the Promotion of Medical Science. ARB acknowledges support from the Department of Pharmacology, Vanderbilt University, and thanks James Weiny for technical assistance with the analysis of recombinant SDR9C7.

Address correspondence to: Takuya Takeichi or Masashi Akiyama, Department of Dermatology, Nagoya University Graduate School of Medicine, 65 Tsurumai-cho, Showa-ku, Nagoya 466-8550, Japan. Phone: 81.52.744.2314; Email: takeichi@med.nagoya-u.ac. jp (T. Takeichi); makiyama@med.nagoya-u.ac.jp (M. Akiyama). Or to: Alan R. Brash, Department of Pharmacology, RRB Room 510, Vanderbilt University, Nashville, Tennessee 37232, USA. Phone: 615.322.3015; Email: alan.brash@vanderbilt.edu.
1. Madison KC. Barrier function of the skin: "la raison d'être" of the epidermis. J Invest Dermatol. 2003;121(2):231-241.

2. Wertz PW. Current understanding of skin biology pertinent to skin penetration: skin biochemistry. Skin Pharmacol Physiol. 2013;26(4-6):217-226.

3. Feingold KR, Elias PM. Role of lipids in the formation and maintenance of the cutaneous permeability barrier. Biochim Biophys Acta. 2014;1841(3):280-294.

4. Akiyama M. Corneocyte lipid envelope (CLE), the key structure for skin barrier function and ichthyosis pathogenesis. J Dermatol Sci. 2017;88(1):3-9.

5. Elias PM, et al. Formation and functions of the corneocyte lipid envelope (CLE). Biochim Biophys Acta. 2014;1841(3):314-318.

6. Takeichi T, Akiyama M. Inherited ichthyosis: Non-syndromic forms. J Dermatol. 2016;43(3):242-251.

7. Marukian NV, et al. Establishing and validating an ichthyosis severity index. J Invest Dermatol. 2017;137(9):1834-1841.

8. Chiba T, Nakahara T, Kohda F, Ichiki T, Manabe M, Furue M. Measurement of trihydroxy-linoleic acids in stratum corneum by tape-stripping: Possible biomarker of barrier function in atopic dermatitis. PLoS ONE. 2019;14(1):e0210013.

9. Hansen HS, Jensen B. Essential function of linoleic acid esterified in acylglucosylceramide and acylceramide in maintaining the epidermal water permeability barrier. Evidence from feeding studies with oleate, linoleate, arachidonate, columbinate and alpha-linolenate. Biochim Biophys Acta. 1985;834(3):357-363.

10. Zheng Y, et al. Lipoxygenases mediate the effect of essential fatty acid in skin barrier formation: a proposed role in releasing omega-hydroxyceramide for construction of the corneocyte lipid envelope. J Biol Chem. 2011;286(27):24046-24056.

11. Dick A, et al. Diminished protein-bound $\omega$-hydroxylated ceramides in the skin of patients with ichthyosis with 12R-lipoxygenase (LOX) or eLOX-3 deficiency. Br J Dermatol. 2017;177(4):e119-e121.

12. Jobard F, et al. Lipoxygenase-3 (ALOXE3) and 12(R)-lipoxygenase (ALOX12B) are mutated in non-bullous congenital ichthyosiform erythroderma (NCIE) linked to chromosome 17p13.1.
Hum Mol Genet. 2002;11(1):107-113.

13. Muñoz-Garcia A, Thomas CP, Keeney DS, Zheng Y, Brash AR. The importance of the lipoxygenase-hepoxilin pathway in the mammalian epidermal barrier. Biochim Biophys Acta. 2014;1841(3):401-408.

14. Krieg P, Fürstenberger G. The role of lipoxygenases in epidermis. Biochim Biophys Acta. 2014;1841(3):390-400.

15. Meguro S, Arai Y, Masukawa Y, Uie K, Tokimitsu I. Relationship between covalently bound ceramides and transepidermal water loss (TEWL). Arch Dermatol Res. 2000;292(9):463-468.

16. Crumrine D, et al. Mutations in recessive congenital ichthyoses illuminate the origin and functions of the corneocyte lipid envelope. JInvest Dermatol. 2019;139(4):760-768.

17. Nemes Z, Marekov LN, Fésüs L, Steinert PM. A novel function for transglutaminase 1: attachment of long-chain omega-hydroxyceramides to involucrin by ester bond formation. Proc Natl Acad Sci U S A. 1999;96(15):8402-8407.

18. Kalinin AE, Kajava AV, Steinert PM. Epithelial barrier function: assembly and structural fea- 
tures of the cornified cell envelope. Bioessays. 2002;24(9):789-800.

19. Elias PM, et al. Basis for the permeability barrier abnormality in lamellar ichthyosis. Exp Dermatol. 2002;11(3):248-256.

20. Pitolli C, et al. Characterization of TG2 and TG1-TG2 double knock-out mouse epidermis. Amino Acids. 2017;49(3):635-642.

21. Ohno Y, Kamiyama N, Nakamichi S, Kihara A. PNPLA1 is a transacylase essential for the generation of the skin barrier lipid $\omega$-O-acylceramide. Nat Commun. 2017;8:14610.

22. Hirabayashi T, et al. PNPLA1 has a crucial role in skin barrier function by directing acylceramide biosynthesis. Nat Commun. 2017;8:14609.

23. Hirabayashi T, Murakami M, Kihara A. The role of PNPLA1 in $\omega$-O-acylceramide synthesis and skin barrier function. Biochim Biophys Acta Mol Cell Biol Lipids. 2019;1864(6):869-879.

24. Grond S, et al. PNPLA1 deficiency in mice and humans leads to a defect in the synthesis of omega-O-acylceramides. J Invest Dermatol. 2017;137(2):394-402.

25. Pichery M, et al. PNPLA1 defects in patients with autosomal recessive congenital ichthyosis and KO mice sustain PNPLA1 irreplaceable function in epidermal omega-O-acylceramide synthesis and skin permeability barrier. Hum Mol Genet. 2017;26(10):1787-1800.

26. Kowalik D, Haller F, Adamski J, Moeller G. In search for function of 2 human orphan SDR enzymes: hydroxysteroid dehydrogenase like 2 (HSDL2) and short-chain dehydrogenase/reductase-orphan (SDR-O). J Steroid Biochem Mol Biol. 2009;117(4-5):117-124.

27. Kallberg Y, Oppermann U, Persson B. Classification of the short-chain dehydrogenase/reductase superfamily using hidden Markov models. FEBS J. 2010;277(10):2375-2386.

28. Zhang $\mathrm{H}$, et al. Quantitative image analysis of protein expression and colocalisation in skin sections. Exp Dermatol. 2018;27(2):196-199.

29. Shigehara Y, et al. Mutations in SDR9C7 gene encoding an enzyme for vitamin A metabolism underlie autosomal recessive congenital ichthyosis. Hum Mol Genet. 2016;25(20):4484-4493.

30. Hotz A, et al. Identification of mutations in SDR9C7 in six families with autosomal recessive congenital ichthyosis. Br J Dermatol. 2018;178(3):e207-e209.
31. Takeichi T, et al. Deficient stratum corneum intercellular lipid in a Japanese patient with lamellar ichthyosis with a homozygous deletion mutation in SDR9C7. Br J Dermatol. 2017;177(3):e62-e64.

32. Lek M, et al. Analysis of protein-coding genetic variation in 60,706 humans. Nature. 2016;536(7616):285-291.

33. Huang W, Solouki S, Koylass N, Zheng SG, August A. ITK signalling via the Ras/IRF4 pathway regulates the development and function of Tr1 cells. Nat Commun. 2017;8:15871.

34. Chiba T, Thomas CP, Calcutt MW, Boeglin WE, O'Donnell VB, Brash AR. The precise structures and stereochemistry of trihydroxy-linoleates esterified in human and porcine epidermis and their significance in skin barrier function: implication of an epoxide hydrolase in the transformations of linoleate. J Biol Chem. 2016;291(28):14540-14554.

35. Yamanashi H, et al. Catalytic activities of mammalian epoxide hydrolases with cis and trans fatty acid epoxides relevant to skin barrier function. J Lipid Res. 2018;59(4):684-695.

36. Sayre LM, Arora PK, Iyer RS, Salomon RG. Pyrrole formation from 4-hydroxynonenal and primary amines. Chem Res Toxicol. 1993;6(1):19-22.

37. Sayre LM, Lin D, Yuan Q, Zhu X, Tang X. Protein adducts generated from products of lipid oxidation: focus on HNE and one. Drug Metab Rev. 2006;38(4):651-675.

38. Hidalgo FJ, Zamora R, Vioque E. Syntheses and reactions of methyl (Z)-9,10-epoxy13-oxo-(E)-11-octadecenoate and methyl (E)-9,10-epoxy-13-oxo-(E)-11-octadecenoate. Chem Phys Lipids. 1992;60(3):225-233.

39. Hidalgo FJ, Zamora R. In vitro production of long chain pyrrole fatty esters from carbonyl-amine reactions. J Lipid Res. 1995;36(4):725-735.

40. Marekov LN, Steinert PM. Ceramides are bound to structural proteins of the human foreskin epidermal cornified cell envelope. J Biol Chem. 1998;273(28):17763-17770.

41. Grond S, et al. Skin barrier development depends on CGI-58 protein expression during late-stage keratinocyte differentiation. JInvest Dermatol. 2017;137(2):403-413.

42. Djian P, Easley K, Green H. Targeted ablation of the murine involucrin gene. J Cell Biol. 2000;151(2):381-388
43. Sevilla LM, et al. Mice deficient in involucrin, envoplakin, and periplakin have a defective epidermal barrier. JCell Biol. 2007;179(7):1599-1612.

44. Candi E, Schmidt R, Melino G. The cornified envelope: a model of cell death in the skin. Nat Rev Mol Cell Biol. 2005;6(4):328-340.

45. Maegawa T, et al. Congenic mapping and candidate gene analysis for streptozotocin-induced diabetes susceptibility locus on mouse chromosome 11. Mamm Genome. 2018;29(3-4):273-280.

46. Teixeira M, et al. Electroporation of mice zygotes with dual guide RNA/Cas9 complexes for simple and efficient cloning-free genome editing. $S c i$ Rep. 2018;8(1):474.

47. Haeussler M, et al. Evaluation of off-target and on-target scoring algorithms and integration into the guide RNA selection tool CRISPOR. Genome Biol. 2016;17(1):148

48. Takeichi T, et al. Phosphorylated signal transducer and activator of transcription 3 in the epidermis in adult-onset Still's disease. J Dermatol. 2017;44(10):1172-1175.

49. Thomas CP, Boeglin WE, Garcia-Diaz Y, O’Donnell VB, Brash AR. Steric analysis of epoxyalcohol and trihydroxy derivatives of 9-hydroperoxy-linoleic acid from hematin and enzymatic synthesis. Chem Phys Lipids. 2013;167-168:21-32.

50. Davis RW, Allweil A, Tian J, Brash AR, Sulikowski GA. Stereocontrolled synthesis of four isomeric linoleate triols of relevance to skin barrier formation and function. Tetrahedron Lett. 2018;59(52):4571-4573.

51. Takeichi T, et al. Biallelic mutations in KDSR disrupt ceramide synthesis and result in a spectrum of keratinization disorders associated with thrombocytopenia. J Invest Dermatol. 2017;137(11):2344-2353.

52. Ohno Y, et al. Essential role of the cytochrome P450 CYP4F22 in the production of acylceramide, the key lipid for skin permeability barrier formation. Proc Natl Acad Sci U S A. 2015;112(25):7707-7712.

53. Ishikawa J, et al. Variations in the ceramide profile in different seasons and regions of the body contribute to stratum corneum functions. Arch Dermatol Res. 2013;305(2):151-162.

54 . Watanabe YS, et al. Anagliptin, a potent dipeptidyl peptidase IV inhibitor: its single-crystal structure and enzyme interactions. JEnzyme Inhib Med Chem. 2015;30(6):981-988. 